\title{
A Diagnosis of Thickness Fluxes in an Eddy-Resolving Model
}

\author{
CARSTEN EDEN \\ Leibniz-Institut für Meereswissenschaften an der Universität Kiel, Kiel, Germany \\ RichaRd J. GREATBATCH \\ Dalhousie University, Halifax, Nova Scotia, Canada \\ JÜRGEN WILLEBRAND \\ Leibniz-Institut für Meereswissenschaften an der Universität Kiel, Kiel, Germany
}

(Manuscript received 21 July 2005, in final form 19 May 2006)

\begin{abstract}
Output from an eddy-resolving model of the North Atlantic Ocean is used to estimate values for the thickness diffusivity $\kappa$ appropriate to the Gent and McWilliams parameterization. The effect of different choices of rotational eddy fluxes on the estimated $\kappa$ is discussed. Using the raw fluxes (no rotational flux removed), large negative values (exceeding $-5000 \mathrm{~m}^{2} \mathrm{~s}^{-1}$ ) of $\kappa$ are diagnosed locally, particularly in the Gulf Stream region and in the equatorial Atlantic. Removing a rotational flux based either on the suggestion of Marshall and Shutts or the more general theory of Medvedev and Greatbatch leads to a reduction of the negative values, but they are still present. The regions where $\kappa<0$ correspond to regions where eddies are acting to increase, rather than decrease (as in baroclinic instability) the mean available potential energy. In the subtropical gyre, $\kappa$ ranges between 500 and $2000 \mathrm{~m}^{2} \mathrm{~s}^{-1}$, rapidly decreasing to zero below the thermocline in all cases. Rotational fluxes and $\kappa$ are also estimated using an optimization technique. In this case, $|\kappa|$ can be reduced or increased by construction, but the regions where $\kappa<0$ are still present and the optimized rotational fluxes also remain similar to a priori values given by the theoretical considerations. A previously neglected component $(\nu)$ of the bolus velocity is associated with the horizontal flux of buoyancy along, rather than across, the mean buoyancy contours. The $\nu$ component of the bolus velocity is interpreted as a streamfunction for eddy-induced advection, rather than diffusion, of mean isopycnal layer thickness, showing up when the lateral eddy fluxes cannot be described by isotropic diffusion only. All estimates show a similar large-scale pattern for $\nu$, implying westward advection of isopycnal thickness over much of the subtropical gyre. Comparing $\nu$ with a mean streamfunction shows that it is about $10 \%$ of the mean in midlatitudes and even larger than the mean in the Tropics.
\end{abstract}

\section{Introduction}

The parameterization for the advective effects of mesoscale activity on tracers in the ocean, as first proposed in the literature by Gent and McWilliams (1990, hereinafter GM), is currently applied in most state-of-theart coarse-resolution ocean general circulation models. In this parameterization, a lateral (thickness) diffusivity has to be specified. In most models, a value of about $1000 \mathrm{~m}^{2} \mathrm{~s}^{-1}$ is used, which sometimes is higher in the

Corresponding author address: Dr. Carsten Eden, IFMGEOMAR, FB I, Düsternbrooker Weg 20, 24105 Kiel, Germany. E-mail: ceden@ifm-geomar.de upper ocean and lower below the thermocline. Because of the sparseness of observations of interior mesoscale activity it appears to be difficult to estimate this parameter directly from available observations. On the other hand, there have been some attempts to infer the thickness diffusivity from the synthetic data of eddy-resolving general circulation models (e.g., Rix and Willebrand 1996; Jochum 1997; Bryan et al. 1999; Treguier 1999; Nakamura and Chao 2000; Roberts and Marshall 2000; Drijfhout and Hazeleger 2001; Peterson and Greatbatch 2001; Solovev et al. 2002).

However, these attempts have only been partly successful even in determining the overall gross magnitude of the thickness diffusivity in the ocean. Rix and Wille-

DOI: $10.1175 / \mathrm{JPO} 2987.1$

(C) 2007 American Meteorological Society 
brand (1996), using 5-yr-averaged moments of mesoscale activity from an eddy-permitting model of the North Atlantic Ocean, suggest that longer time series are needed to get stable estimates. Jochum (1997), using 20-yr averages of eddy fluxes from the same model, came to the same conclusion. Both were only able to give a rough estimate of mean thickness diffusivity in the southern part of the subtropical North Atlantic thermocline, excluding western boundary current regions.

Bryan et al. (1999) pointed out that it might be necessary to subtract (or add) rotational eddy fluxes from the "raw" eddy fluxes to get meaningful results in terms of thickness diffusivity. Even with perfect statistics of the mesoscale activity in the real ocean, one might still obtain incorrect results without considering rotational fluxes, making the task of determining the value of the "real" thickness diffusivity for use in a general circulation model very difficult.

On the other hand, several authors (Roberts and Marshall 2000; Drijfhout and Hazeleger 2001; Jayne and Marotzke 2002) perform a Helmholtz decomposition of the eddy fluxes into divergent and rotational parts. Using this decomposition, however, it is assumed that the part of the fluxes that can be used for eddy parameterizations is completely irrotational, that is, an overly restrictive assumption not imposed, for example, in Eden et al. (2007). Moreover, the Helmholtz decomposition depends on the definition of boundary conditions for divergent and rotational parts, which cannot be defined in a unique manner. This choice for rotational fluxes remains therefore ambiguous; see also the discussion in Allen et al. (1997) and Fox-Kemper et al. (2003). Nakamura and Chao (2000) try to infer patterns of thickness diffusivity in an eddy-permitting model of the North Atlantic by considering the divergence of the eddy fluxes only, which eliminates the problem of rotational fluxes by construction, but yields rather noisy results and no clear sign or order of magnitude of the thickness diffusivity.

Using data from a realistic eddy-resolving model of the North Atlantic, we test two choices for the rotational fluxes-one proposed by Marshall and Shutts (1981), and applied by Marshall (1984), and a more general approach by Medvedev and Greatbatch (2004). As an alternative to specifying a rotational flux based on theoretical considerations, we also estimate rotational fluxes using minimizations with respect to the value of thickness diffusivity, but including information about rotational fluxes. In all our estimates we show the thickness diffusivity $(\kappa)$, related to the horizontal eddy flux of buoyancy down the horizontal gradient of mean buoyancy, and a new parameter $(\nu)$, related to a previ- ously neglected component of the eddy flux along, rather than across, the mean buoyancy contours. We argue that this part of the flux, which is not included in GM, might also be important for large-scale flows.

Following this introduction, we present and discuss the GM parameterization in section 2 and show eddy fluxes from an eddy-resolving realistic model of the North Atlantic in section 3. In section 4, we review the rotational eddy flux choice of Marshall and Shutts (1981) and show thickness diffusivity $\kappa$ and $\nu$ using this rotational flux and using the raw eddy fluxes. In section 5 , we introduce the rotational flux of Medvedev and Greatbatch (2004) and show its consequences on $\kappa$ and $\nu$, followed by section 6 in which we introduce a minimal condition to constrain $\kappa, \nu$, and the rotational flux and show results from different minimizations. The last section summarizes and discusses our conclusions.

\section{A generalization of the GM parameterization}

In this section we briefly derive the GM parameterization in the framework of the transformed Eulerian mean (Andrews and McIntyre 1976). For simplicity, we neglect any complication from a nonlinear equation of state and use $b$ as buoyancy, with $b=-g\left(\rho-\rho_{0}\right) / \rho_{0}$. The mean buoyancy budget is given by

$$
\bar{b}_{t}+\overline{\mathbf{u}} \cdot \boldsymbol{\nabla} \bar{b}+\boldsymbol{\nabla} \cdot \mathbf{F}=\bar{Q},
$$

with buoyancy $b=\bar{b}+b^{\prime}$, velocity $\mathbf{u}=\overline{\mathbf{u}}+\mathbf{u}^{\prime}$, and $\bar{Q}$ being the mean diabatic forcing; $\mathbf{F}=\overline{\mathbf{u}^{\prime} b^{\prime}}$ denotes the eddy buoyancy flux, and primes are deviations from a temporal mean denoted by an overbar. Now we decompose $\mathbf{F}$ as

$$
\mathbf{F}=-K \nabla \bar{b}+\mathbf{B} \times \nabla \bar{b}+\boldsymbol{\nabla} \times \boldsymbol{\theta},
$$

with a diapycnal diffusivity $K=-|\nabla \bar{b}|^{2}(\mathbf{F}-\nabla \times$ $\boldsymbol{\theta}) \cdot \nabla \bar{b}$ and a rotational flux given by the vector potential $\boldsymbol{\theta}$, which drops out in the divergence of $\mathbf{F}$ and therefore does not show up in Eq. (1). Following GM, we assume for now, and the remainder of this paper, that mesoscale activity is completely adiabatic so that $K=0$. Note that this view of the effect of eddy activity in the ocean has been recently under debate (see, e.g., Radko and Marshall 2004; Eden et al. 2007). We also begin by ignoring the rotational vector potential, so $\boldsymbol{\theta}=0$ in this section. Note, however, that we will define certain nonzero vector potentials $\boldsymbol{\theta}$ in the following sections for reasons explained below. Since

$$
\boldsymbol{\nabla} \cdot \boldsymbol{F}=\boldsymbol{\nabla} \times \mathbf{B} \cdot \boldsymbol{\nabla} \bar{b} \equiv \mathbf{u}^{*} \cdot \boldsymbol{\nabla} \bar{b},
$$

the curl of $\mathbf{B}$ acts like an eddy-induced, three-dimensional advection velocity $\mathbf{u}^{*}$, which adds to the Eulerian mean velocity $\overline{\mathbf{u}}$ in Eq. (1) and is often called the "bolus velocity"; we will stick to this terminology [see, how- 
a)

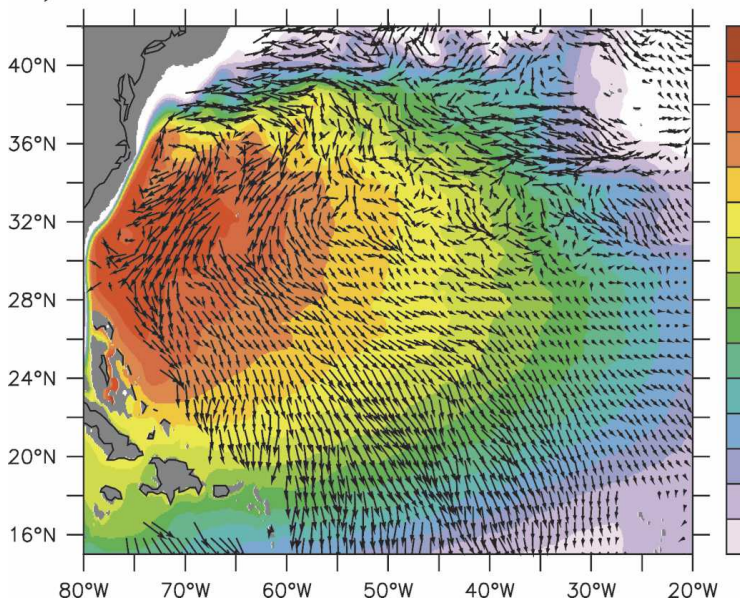

b)

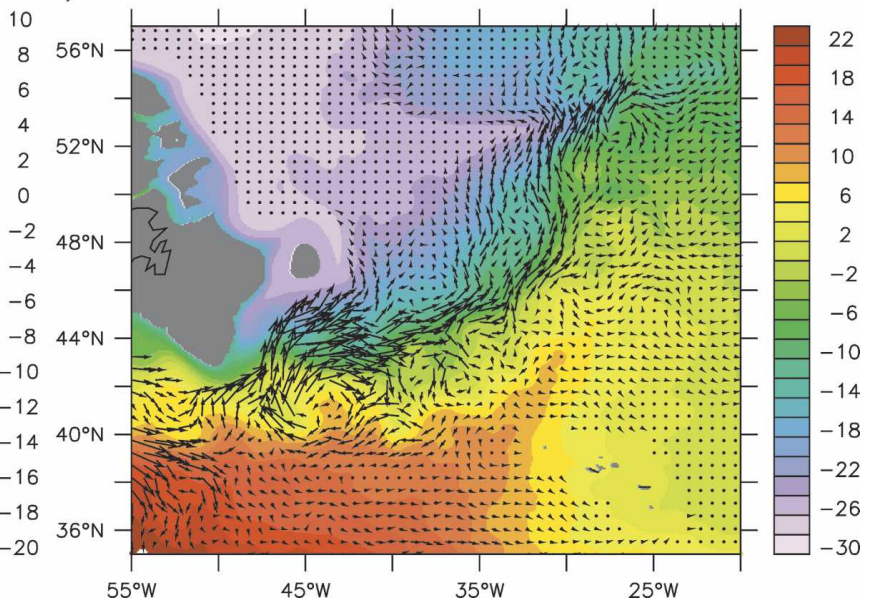

FIG. 1. Horizontal eddy fluxes $\left(\overline{\mathbf{u}_{h}^{\prime} b^{\prime}}\right.$; arrows) and mean buoyancy $\bar{b}$ (shading; $\left.\mathrm{m} \mathrm{min}^{-2}\right)$ in an eddy-resolving model of the North Atlantic $(1 / 12$ resolution $)$ in 300-m depth. Shown are 5-yr averages for two different regions. The eddy fluxes are smoothed with a Hanning window over $3^{\circ} \times 3^{\circ}$; vector magnitudes are limited to the maximal length shown in the figure.

ever, McDougall and McIntosh (2001), who argue that this term strictly should be called "quasi-Stokes drift"]. The sum of bolus velocity and Eulerian mean velocity is sometimes called the "residual velocity."
The second part of the flux decomposition in Eq. (2) introduces a vector streamfunction $\boldsymbol{B}$ for the bolus velocity $\mathbf{u}^{*}$, which can be written as (using the gauge condition $\mathbf{B} \cdot \boldsymbol{\nabla} \bar{b}=0$ )

$$
\mathbf{B}=-|\nabla \bar{b}|^{-2} \mathbf{F} \times \nabla \bar{b}=|\nabla \bar{b}|^{-2}\left(\begin{array}{l}
\overline{v^{\prime} b^{\prime}} \bar{b}_{z}-\overline{w^{\prime} b^{\prime}} \bar{b}_{y} \\
\overline{w^{\prime} b^{\prime}} \bar{b}_{x}-\overline{u^{\prime} b^{\prime}} \bar{b}_{z} \\
\overline{u^{\prime} b^{\prime} b_{y}}-\overline{v^{\prime} b^{\prime} \bar{b}_{x}}
\end{array}\right) \approx \bar{b}_{z}^{-1}\left(\begin{array}{c}
\overline{v^{\prime} b^{\prime}} \\
\overline{u^{\prime} b^{\prime}} \\
\overline{u^{\prime} b^{\prime} s_{2}}-\overline{v^{\prime} b^{\prime} s_{1}}
\end{array}\right)
$$

where $\mathbf{s}=\left(s_{1}, s_{2}\right)^{\mathrm{T}}=\bar{b}_{z}^{-1} \boldsymbol{\nabla}_{h} \bar{b}$ denotes the isopycnal slope vector. Note that the last approximation in Eq. (4) is valid only for $\left|\bar{b}_{z}\right| \gg\left|\nabla_{h} \bar{b}\right|$ (we care about this situation only and ignore complications in, e.g., a surface mixed layer).

In Eq. (4), the streamfunction $\mathbf{B}$ is expressed in terms of the eddy buoyancy fluxes, which have to be parameterized for use in a coarse-resolution model. In the GM parameterization this is achieved by assuming that the horizontal eddy fluxes $\overline{\mathbf{u}_{h}^{\prime} b^{\prime}}$ are directed down the horizontal gradient of the mean buoyancy, that is, $\overline{\mathbf{u}_{h}^{\prime} b^{\prime}} \approx-\kappa \bar{\nabla}_{h} b$, with $\kappa>0$. However, as discussed below, an inspection of the eddy fluxes in a numerical model (cf. Fig. 1) shows that the flux $\overline{\mathbf{u}_{h}^{\prime} b^{\prime}}$ is not everywhere down the mean buoyancy gradient, that is, it tends to show a significant component perpendicular to the mean buoyancy gradient, in particular, in regions of strong currents. Therefore, we express the eddy fluxes as

$$
\mathbf{F}_{h} \equiv \overline{\mathbf{u}_{h}{ }_{h} b^{\prime}}=-\kappa \nabla_{h} \bar{b}-\nu \nabla \bar{\gamma} \bar{b}
$$

with $\kappa=-\left|\nabla_{h} \bar{b}\right|^{-2} \mathbf{F}_{h} \cdot \boldsymbol{\nabla}_{h} \bar{b}$ and $\nu=-\left|\nabla_{h} \bar{b}\right|^{-2} \mathbf{F}_{h} \cdot \boldsymbol{\nabla} \bar{b}$. The operator $\boldsymbol{\nabla}$ is given by $\boldsymbol{\nabla}=(-\partial / \partial y, \partial / \partial x)^{\mathrm{T}}$, that is, a shorthand for $\mathbf{k} \times \boldsymbol{\nabla}_{h}$ (the vector subscript $\urcorner$ shall denote anticlockwise rotation of a horizontal vector by $90^{\circ}$ ). Note that the flux decomposition in Eq. (5) is always possible as long as $\left|\nabla_{h} \bar{b}\right| \neq 0$. It should also be noted that $\mathbf{B}$, and consequently $\kappa$ and $\nu$, depend on the choice of the rotational vector potential $\boldsymbol{\theta}$ (which we have set to zero for now), an issue explored below.

The flux decomposition equation (5) is equivalent to

$$
\mathbf{F}_{h}=-\mathbf{K} \nabla_{h} \bar{b} \text { with } \mathbf{K}=\left(\begin{array}{cc}
\kappa & -\nu \\
\nu & \kappa
\end{array}\right)=\underbrace{\left(\begin{array}{cc}
\kappa & 0 \\
0 & \kappa
\end{array}\right)}_{\text {symm. part }}+\underbrace{\left(\begin{array}{cc}
0 & -\nu \\
\nu & 0
\end{array}\right)}_{\text {antisymm. part }},
$$


introducing the diffusion tensor $\mathbf{K}$, which is composed of a symmetric part and an antisymmetric (skew) part. The symmetric part of $\mathbf{K}$ is related to (isotropic) alonggradient diffusion (as in GM) while the antisymmetric (skew) part is related to advection, as discussed below. Note that since in principle four components of $\mathbf{K}$ have to be specified with only two components of $\mathbf{F}_{h}$, for a diagnostic purpose as discussed here, other forms for $\mathbf{K}$ are also possible or convenient. For instance, Smith and Gent (2004) introduce an anisotropic diffusion tensor that allows for different diffusivities in two principal directions to be specified. They propose to use the direction of the mean flow, that is, to have different diffusivities in along-stream and cross-stream directions. The motivation comes from numerical considerations since the form of Smith and Gent (2004) allows weak or zero mixing in the cross-stream direction.

On the other hand, choosing the decomposition equation (5) so that the diffusion tensor is given by Eq. (6), the diffusivity $\kappa$ has a clear physical meaning, as shown below, considering the energy budget, while the remainder, that is, the $\nu$-related part of the eddy flux $\overline{\mathbf{u}_{h}^{\prime} b^{\prime}}$, has an advective character (see below), shows up when the lateral eddy fluxes cannot be described by isotropic diffusion only and does not figure in the integral energy budget (see below).

Using the expression, Eq. (5), we obtain for the streamfunction of the bolus velocity in Eq. (4)

$$
\mathbf{B}=\kappa\left(\begin{array}{c}
-s_{2} \\
s_{1} \\
0
\end{array}\right)+\nu\left(\begin{array}{c}
-s_{1} \\
-s_{2} \\
s_{2}^{2}+s_{1}^{2}
\end{array}\right),
$$

while the bolus velocity $\mathbf{u}^{*}$ itself is given by

$$
\begin{aligned}
\boldsymbol{\nabla} \times \mathbf{B}= & \left(\begin{array}{c}
-\left(\kappa s_{1}\right)_{z} \\
-\left(\kappa s_{2}\right)_{z} \\
\left(\kappa s_{1}\right)_{x}+\left(\kappa s_{2}\right)_{y}
\end{array}\right)+\left(\begin{array}{c}
\left(\nu s_{2}\right)_{z} \\
-\left(\nu s_{1}\right)_{z} \\
\left(-\nu s_{2}\right)_{x}+\left(\nu s_{1}\right)_{y}
\end{array}\right) \\
& +\left(\begin{array}{c}
\frac{\partial}{\partial y}\left(\nu s_{2}^{2}+\nu s_{1}^{2}\right) \\
-\frac{\partial}{\partial x}\left(\nu s_{2}^{2}+\nu s_{1}^{2}\right) \\
0
\end{array}\right)
\end{aligned}
$$

It is the first term on the rhs of Eq. (8) that is added in GM to the mean velocity $\overline{\mathbf{u}}$ in coarse-resolution models to advect tracers. The parameter $\kappa$ denotes thickness diffusivity and is usually chosen of the order of 1000 $\mathrm{m}^{2} \mathrm{~s}^{-1}$. The assumption that $\kappa$ is positive ensures that the parameterization releases available potential energy from the mean state (Gent et al. 1995) (see also below). Note also that the third term on the rhs of Eq.
(8) will be much smaller than the other two for small isopycnal slopes.

\section{Interpretation of the GM parameterization}

What is the meaning of the parameter $\nu$ and its related part of the bolus velocity? That part of the bolus velocity associated with $\nu$ does not figure in the original GM parameterization, and is a new aspect of the bolus velocity, even though it emerges quite naturally from our analysis. Some insight into the nature of the parameter $\nu$ can be gained by considering the time-averaged buoyancy perturbation budget in the quasigeostrophic approximation

$$
\bar{b}_{t}+\nabla_{h} \cdot\left(\overline{\mathbf{u}}_{g} \bar{b}\right)+R_{z} \bar{w}=-\nabla_{h} \cdot \mathbf{F}_{g},
$$

where the geostrophic, zero-order velocities are given by the streamfunction $\mathbf{u}_{g}=\boldsymbol{\nabla} \psi$, the background stratification by $R(z)$, and the (horizontal) eddy fluxes by $\mathbf{F}_{g}=\overline{\mathbf{u}_{g}^{\prime} b^{\prime}}$. In accordance to the quasigeostrophic approximation, we have decomposed the full buoyancy as $R(z)+b$ and the perturbation buoyancy $b$ again into time average and perturbation as $b=\bar{b}+b^{\prime}$. The diabatic forcing $Q$ has been neglected in Eq. (9) and for $\mathbf{F}_{g}$ the above flux decomposition given by Eq. (5) is used again,

$$
\bar{b}_{t}+\overline{\mathbf{u}}_{g} \cdot \boldsymbol{\nabla}_{h} \bar{b}-\nabla_{\urcorner} \nu \cdot \nabla_{h} \bar{b}-\nabla_{h} \cdot \kappa \boldsymbol{\nabla} \bar{b}+R_{z} \bar{w}=0 .
$$

The density budget Eq. (10) can be transformed into a budget of mean isopycnal layer thickness $\bar{h}$,

$$
\bar{h}_{t}+\nabla_{\uparrow} \bar{\psi} \cdot \nabla_{h} \bar{h}+\nabla_{\uparrow} \nu \cdot \nabla_{h} \bar{h}+\bar{w}_{z}=\nabla_{h} \cdot \kappa \nabla_{h} \bar{h},
$$

where $\bar{h}$ is given by

$$
\bar{h}=\frac{\partial}{\partial z}\left(\frac{\bar{b}+R}{R_{z}}\right)
$$

and where it was assumed that $\kappa$ and $\nu$ are independent of depth [note that Gent et al. (1995) also need to assume that $\partial \kappa / \partial z=0$ to relate $\kappa$ to thickness diffusion in their parameterization]. It becomes obvious that there is eddy-induced diffusion of mean thickness related to the downgradient part of $\mathbf{F}_{g}$ and given by the term $\boldsymbol{\nabla}_{h} \cdot \kappa \boldsymbol{\nabla}_{h} \bar{h}$ in Eq. (11); therefore $\kappa$ is often called thickness diffusivity. In addition, however, there is eddyinduced advection of mean thickness related to the part of $\mathbf{F}_{g}$ circulating along contours of $\bar{b}$ and given by the term $\boldsymbol{\nabla} \nu \cdot \nabla_{h} \bar{h}$. Note that $\nu$ acts like a streamfunction, which adds to the mean geostrophic streamfunction $\bar{\psi}$. Thus, the physical meaning of the parameter $\nu$ is that it 
is a streamfunction for eddy advection of mean isopycnal thickness. ${ }^{1}$ This effect is complementary to eddy diffusion of thickness (related to $\kappa$ ) and shows up when the lateral eddy fluxes cannot be described by isotropic diffusion only.

Next we show that in the eddy variance balance of $b$, which gives the budget of (available) eddy potential energy in the quasigeostrophic approximation, the downgradient flux related to $\kappa$ shows up as a dissipation of eddy potential energy (as long as $\kappa$ is positive), while $\nu$ does not figure in that equation. Eddy potential energy in the quasigeostrophic approximation is $\bar{\phi} / R_{z}$, where the eddy variance $\bar{\phi}=\overline{b^{\prime 2}} / 2$ is given by

$$
\bar{\phi}_{t}+\nabla_{h} \cdot \overline{\mathbf{u}_{h} \phi}=\kappa\left|\nabla_{h} \bar{b}\right|^{2}-R_{z} \overline{b^{\prime} w^{\prime}} .
$$

Again, we have set $Q=0$ and have used the flux decomposition Eq. (5). Integrating over a closed domain, the advective terms in Eq. (12) drop out and an integral constraint on $\kappa$ is left, while $\nu$ does not figure in that equation. Therefore, there is no effect on eddy potential energy associated with the $\nu$-related part of the eddy fluxes, which means, in turn, that there is no integral constraint on the sign of $\nu$ as has been argued for thickness diffusivity $\kappa$.

Neglecting the time tendency and the flux of variance in Eq. (12) yields a local relation between the thickness diffusivity $\kappa$ and $\overline{b^{\prime} w^{\prime}}$. A similar, but exact result is obtained, following Eden et al. (2007), by introducing a rotational flux in the flux decomposition, that is, $\mathbf{F}_{g}=$ $-\boldsymbol{\kappa} \boldsymbol{\nabla}_{h} \bar{b}-\nu \nabla \bar{b}+\boldsymbol{\nabla} \theta_{q g}$. Note that the rotational flux $\nabla \theta_{q g}$ drops out, taking the divergence in Eq. (9), but does show up in the variance equation for $\bar{\phi}$. Eden et al. (2007) use the full hierarchy of budgets of moments of $b^{\prime}$ [their generalized transformed residual mean (TRMG) case], which can be used to write the following local expression for $\kappa$ :

$$
\begin{aligned}
\kappa\left|\nabla_{h} \bar{b}\right|^{2} R_{z}^{-1}= & \overline{b^{\prime} w^{\prime}}-\frac{1}{2} \mathcal{D}\left(\overline{b^{\prime 2} w}\right)+\frac{1}{3 !} \mathcal{D}^{2}\left(\overline{b^{\prime 3} w}\right) \\
& -\frac{1}{4 !} \mathcal{D}^{3}\left(\overline{b^{\prime 4} w}\right)+\cdots
\end{aligned}
$$

with the operator $\mathcal{D}(\cdots)=\boldsymbol{\nabla}_{h} \cdot \boldsymbol{\nabla}_{h} \bar{b}\left|\boldsymbol{\nabla}_{h} \bar{b}\right|^{-2}(\cdots)$ and a similar expansion for the streamfunction $\nu$ [the time tendency of $\phi$ has been neglected in Eq. (13)]. Thus, correlations between vertical velocity and density are related to the thickness diffusivity $\kappa$. The leading term in the expression, $\overline{b^{\prime} w^{\prime}}$, appears in the eddy potential

\footnotetext{
${ }^{1}$ Note that this interpretation is similar to that of Roberts and Marshall (2000), who consider the effect of the part of the eddy tracer fluxes perpendicular to gradients of the mean tracer as being akin to advection of mean tracer extrema in the horizontal plane (cf. their Fig. 8 and the discussion).
}

energy budget Eq. (12) and is related to baroclinic instability (Lorenz, 1955). We show below that in the numerical model this term is usually negative, indicating the release of eddy potential energy by the eddies and implying positive $\kappa$, but that regions exist where $\overline{b^{\prime} w^{\prime}}$ is positive, indicating that eddies are acting to increase the mean potential energy, implying negative $\kappa$ [given that higher-order terms in Eq. (13) can be neglected].

Note that we use the quasigeostrophic approximation here only to connect to available potential energy. Transforming the averaged density budget, Eq. (1), using the flux decomposition, Eq. (5), and the continuity equation to a system using $\bar{b}$ as vertical coordinate also shows the advective nature of the parameter $\nu$ and the diffusive nature of the parameter $\kappa$. However, to make the analogy complete one has also to assume that $\kappa$ and $\nu$ are independent of depth. Thus, for the general case, the $\kappa$ - and $\nu$-related fluxes are acting "like" thickness diffusion and advection but are not identical.

\section{Eddy fluxes in the eddy-resolving model}

To illustrate how well the Fickian assumption $\overline{\mathbf{u}_{h}^{\prime} b^{\prime}} \approx$ $-\boldsymbol{\kappa} \overline{\boldsymbol{\nabla}}_{h} b$ assumed by GM actually holds up, we show $\overline{\mathbf{u}_{h}^{\prime} b^{\prime}}$ and $\bar{b}$ in the main thermocline in a realistic eddyresolving model $\left({ }^{1} /{ }^{\circ}\right)$ of the North Atlantic in Fig. 1. The horizontal resolution of this model is about $10 \mathrm{~km}$ at the equator decreasing to about $5 \mathrm{~km}$ in high latitudes. The model domain extends from $20^{\circ} \mathrm{S}$ to $70^{\circ} \mathrm{N}$ with open boundaries (Stevens 1990) at the northern and southern boundaries and with a restoring zone in the Eastern Mediterranean. There are 45 vertical geopotential levels with increasing thickness with depth, ranging from $10 \mathrm{~m}$ at the surface to $250 \mathrm{~m}$ near the maximal depth of $5500 \mathrm{~m}$. The model is based on a rewritten version (the numerical code, together with all configurations used in this study, can be accessed online at http://www.ifm.uni-kiel.de/fb/fb1/tm/data/pers/ ceden/spflame/index.html) of the second Modular Ocean Model (MOM2) (Pacanowski 1995), similar to the one used in Eden and Böning (2002) and Brandt et al. (2007) and identical to the one used in Dengler et al. (2004). All moments shown here are averaged over 5 yr following a spinup phase of 10-yr model integration using climatological surface forcing, which is identical to the forcing used in Eden and Böning (2002) and Dengler et al. (2004). For each of the five years, four seasonal means of $\overline{\mathbf{u}_{h}^{\prime} b^{\prime}}\left(\overline{w^{\prime} b^{\prime}}\right.$, etc.) have been summed up to remove the seasonal cycle.

Figure 1a displays the situation in the subtropical gyre at 300-m depth. Here, the eddy fluxes appear indeed to be down the gradient of $\bar{b}$. There is a bowl- 
shaped maximum of buoyancy near the western boundary, and south of the Gulf Stream area the eddy fluxes $\overline{\mathbf{u}_{h}^{\prime} b^{\prime}}$ point outward of that maximum. In the Gulf Stream area itself, however, the eddy fluxes appear to be along contours of constant $\bar{b}$. This behavior is even more clear in the area of the North Atlantic Current (Fig. 1b) where the dominant direction of the eddy fluxes appears to be, as well, more perpendicular to $\boldsymbol{\nabla}_{h} \bar{b}$. Moreover, the (smaller) downgradient part of the flux shows no clear sign as in the subtropical gyre.

However, even if the fluxes do not appear to be downgradient, this does not mean that the GM parameterization is incorrect. As noted at the beginning of section 2 , it is possible to add (or remove) a rotational flux $\theta$ to the eddy buoyancy fluxes. This rotational flux is not affecting the mean density budget, but is affecting the definition of the parameter $\kappa$ and $\nu$ in Eq. (5). It might be possible to remove a rotational flux from $\mathbf{F}$ such that the downgradient GM parameterization remains valid. In the following we will test two choices for such a rotational flux coming from theoretical considerations and also an optimization technique to determine the rotational flux.

\section{Rotational eddy fluxes: Marshall and Shutts (1981)}

A choice for rotational eddy fluxes was proposed by Marshall and Shutts (1981). They consider the eddy variance equation in the quasigeostrophic approximation [similar to Eq. (12)] in which they assume a steadystate and adiabatic flow regime,

$$
\boldsymbol{\nabla}_{h} \cdot \overline{\mathbf{u}_{h} \phi}+\mathbf{F}_{h} \cdot \nabla_{h} \bar{b}+R_{z} \overline{b^{\prime} w^{\prime}} \approx 0,
$$

where $\bar{\phi}=\overline{b^{\prime 2} / 2}$ denotes, as before, eddy variance. They further neglect advection of $\phi$ by the perturbation flow $\left(\overline{\mathbf{u}_{h}^{\prime} \phi}\right)$ and assume that the mean flow $\overline{\mathbf{u}}_{h}$ can be described by a (geostrophic) streamfunction $(\psi)$ and flows parallel to the mean buoyancy $(\bar{b})$ contours so that $\overline{\mathbf{u}}_{h}=\nabla \psi$ with $\psi=\psi(\bar{b}, z){ }^{2}$ Specifically, they assume that

$$
\overline{\mathbf{u}_{h} \phi}=\overline{\mathbf{u}}_{h} \bar{\phi}=\bar{\phi}\left(\frac{\partial \psi}{\partial b}\right)_{\urcorner} \bar{b} \equiv \gamma \underset{\urcorner}{\nabla} \bar{b},
$$

where $\gamma=\bar{\phi} \partial \psi / \partial b$, with the implication that the flux of variance flows along contours of mean buoyancy $\bar{b}$. Now, expressing the eddy flux as $\mathbf{F}_{h}=-\kappa \boldsymbol{\nabla}_{h} \bar{b}-\nu \boldsymbol{\nabla} \bar{b}+$ $\boldsymbol{\nabla} \theta$, and using this and expression Eq. (15) in the eddy variance budget, Eq. (14), yields

\footnotetext{
${ }^{2}$ The dependency of $\psi$ on $\bar{b}$ is clear since the flow follows $\bar{b}$ contours. However, this dependency on $b$ might change with depth; therefore $\psi=\psi(\bar{b}, z)$.
}

$$
\overline{w^{\prime} b^{\prime}} R_{z}+\left(\underset{\eta}{\theta} \theta-\nabla_{\urcorner} \gamma\right) \cdot \nabla_{h} \bar{b}=\kappa\left|\nabla_{h} \bar{b}\right|^{2},
$$

which ultimately motivates one to choose $\gamma=\theta$. Note that by this choice the thickness diffusivity $\kappa$ is proportional to $\overline{w^{\prime} b^{\prime}}$ (in this approximation), a relation that we diagnose from our model results below, which we already noted in section 2 .

Since Eq. (16) can also be written as

$$
\overline{w^{\prime} b^{\prime}} R_{z}+\left[\underset{\urcorner}{\nabla} \theta-\left(\frac{\partial \psi}{\partial b}\right) \underset{\urcorner}{\bar{\phi}}\right] \cdot \nabla_{h} \bar{b}=\kappa\left|\nabla_{h} \bar{b}\right|^{2},
$$

it is easy to see that the choice of Marshall and Shutts (1981) is equivalent to the assumption that the rotational part of the eddy flux $\mathbf{F}_{h}$ is a part of $\mathbf{F}_{h}$ circulating along contours of $\bar{\phi}$. This assumption is supported looking again at the eddy fluxes in the North Atlantic Current region. Figure 2 a displays the eddy fluxes $\mathbf{F}$ and the eddy variance $\bar{\phi}$ in that region. It is indeed evident in this region with high levels of eddy variance that a large part of the eddy flux is circulating along contours of $\bar{\phi}$, consistent with the choice of Marshall and Shutts (1981). Figure $2 \mathrm{~b}$ shows the fluxes of variance $\overline{\mathbf{u}_{h} \phi}$, together with the mean buoyancy $\bar{b}$. In agreement to the assumption Eq. (15), most of $\overline{\mathbf{u}_{h} \phi}$ flows parallel to $\bar{b}$ contours.

Following Marshall and Shutts (1981), we define, in a first attempt, the rotational part $\left(\mathbf{F}_{R}\right)^{3}$ of the eddy fluxes $\mathbf{F}_{h}$ as

$$
\mathbf{F}_{R}=\left(\frac{\partial \psi}{\partial b}\right)_{\urcorner} \bar{\phi} \text { with } \frac{\partial}{\partial b} \psi=\bar{\phi}^{-1}\left|\nabla_{h} \bar{b}\right|^{-2} \overline{\mathbf{u}_{h} \phi} \cdot \underset{7}{\nabla} \bar{b},
$$

where the last expression comes from the assumption, Eq. (15). We subtract $\mathbf{F}_{R}$ from the raw fluxes $\mathbf{F}$ to obtain the streamfunction B. For this choice, the thickness diffusivity $\kappa$ and $\nu$ are given by

$$
\begin{aligned}
& \kappa_{\mathrm{MS}}=-\left|\boldsymbol{\nabla}_{h} \bar{b}\right|^{-2}\left[\overline{\mathbf{u}_{h}^{\prime} b^{\prime}}-\left(\frac{\partial \psi}{\partial b}\right)_{\urcorner} \bar{\phi}\right] \cdot \nabla_{h} \bar{b} \quad \text { and } \\
& \nu_{\mathrm{MS}}=-\left|\boldsymbol{\nabla}_{h} \bar{b}\right|^{-2}\left[\overline{\mathbf{u}_{h}^{\prime} b^{\prime}}-\left(\frac{\partial \psi}{\partial b}\right)_{\urcorner} \bar{\phi}\right] \cdot \boldsymbol{\nabla}_{\urcorner} \bar{b},
\end{aligned}
$$

where we have labeled the parameters $\kappa$ and $\nu$ accordingly to indicate that they are derived using Eq. (19) and Eq. (20).

\footnotetext{
${ }^{3}$ Strictly speaking, $\mathbf{F}_{R}$ is only the horizontal component of the rotational flux. A vertical component of the rotational flux is needed to cancel the horizontal divergence of $\mathbf{F}_{R}$. Only if, as assumed by Marshall and Shutts (1981), $\partial \psi / \partial b$ is horizontally constant, the vertical component of the rotational flux can be set to zero. In that case, the flux potential $\boldsymbol{\theta}$ is given by $\boldsymbol{\theta}=(0,0,-\gamma)^{\mathrm{T}}$ and $(\boldsymbol{\nabla} \times \boldsymbol{\theta})_{h}=\boldsymbol{\nabla} \gamma$.
} 

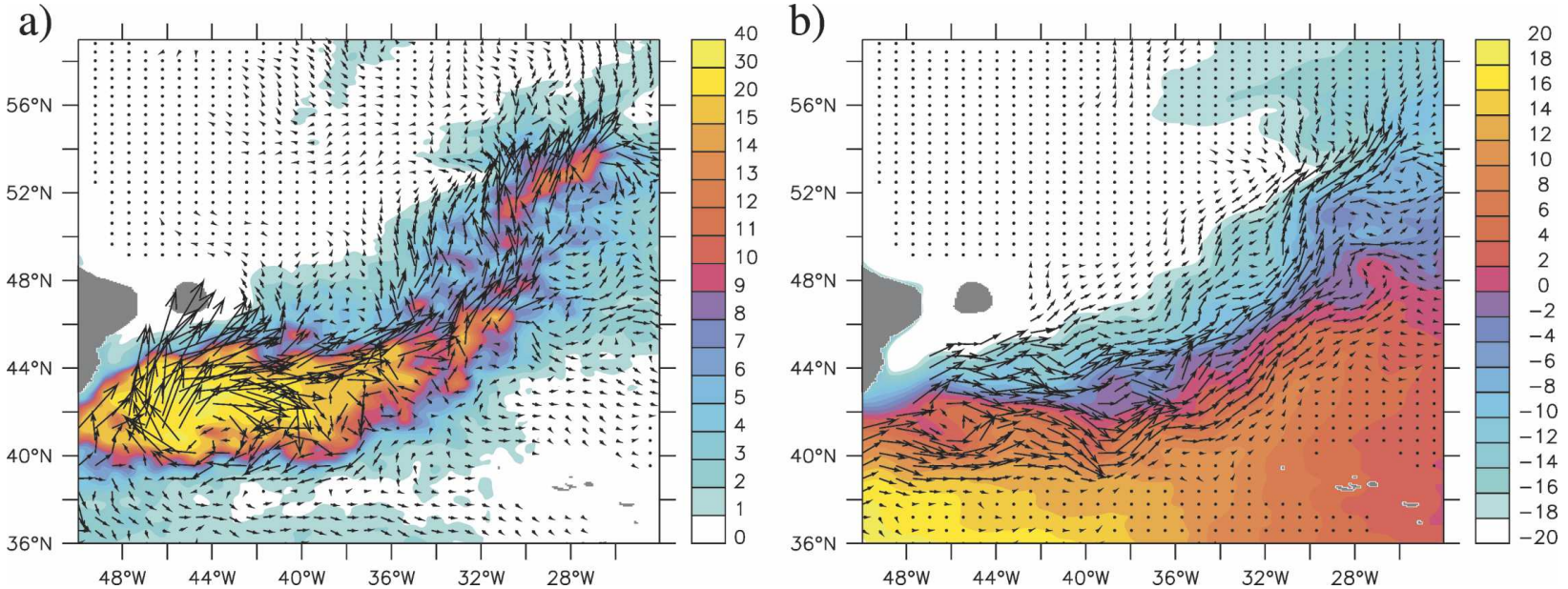

FIG. 2. (a) Horizontal eddy fluxes $\left(\overline{\mathbf{u}_{h}^{\prime} b^{\prime}} \text {; arrows) and eddy variance } \bar{\phi} \text { [shading; (m min }{ }^{-2}\right)^{2}$ ] in 300-m depth. (b) Horizontal fluxes of variance $\left(\overline{\mathbf{u}_{h} \phi}\right.$; arrows) and mean buoyancy $\bar{b}$ (shading; $\left.\mathrm{m} \mathrm{min}^{-2}\right)$ in the same region. Shown are 5 -yr averages for two different regions; $\overline{\mathbf{u}_{h}^{\prime} b^{\prime}}$ and $\overline{\mathbf{u}_{h} \phi}$ are spatially averaged over 12 grid points.

Now we consider the actual thickness diffusivities $\kappa$ and their counterpart $\nu$ (which is not included in the GM parameterization) as diagnosed from the eddyresolving model. We start with $\kappa$ and $\nu$ calculated from the "raw" eddy fluxes, that is, taking $\boldsymbol{\theta}=\mathbf{0}$ as

$$
\begin{aligned}
& \kappa_{\text {raw }}=-\left|\nabla_{h} \bar{b}\right|^{-2} \overline{\mathbf{u}_{h}^{\prime} b^{\prime}} \cdot \nabla_{h} \bar{b} \text { and } \\
& \nu_{\text {raw }}=-\left|\nabla_{h} \bar{b}\right|^{-2} \overline{\mathbf{u}_{h}^{\prime} b^{\prime}} \cdot \nabla_{\urcorner} \bar{b},
\end{aligned}
$$

where we have again labeled the parameters $\kappa$ and $\nu$ to indicate that they are derived from Eq. (21).

Figure 3 shows $\kappa_{\text {raw }}$ and $\nu_{\text {raw }}$ over the North Atlantic in the main thermocline. In the subtropics, distant from any boundary currents, $\kappa_{\text {raw }}$ is indeed positive with values of $500-1500 \mathrm{~m}^{2} \mathrm{~s}^{-1}$, thus close to the canonical value of about $1000 \mathrm{~m}^{2} \mathrm{~s}^{-1}$ used in coarse-resolution models. Note that this is the region in which Rix and Willebrand (1996) estimated a thickness diffusivity of about $1000 \mathrm{~m}^{2} \mathrm{~s}^{-1}$ in their eddy-permitting model. It appears that higher horizontal resolution and more vigorous eddy activity in our eddy-resolving model yields nevertheless similar thickness diffusivities as in Rix and Willebrand (1996) for the subtropical gyre. We note in passing that taking simple 5-yr averages of all model results, without removing the seasonal cycle, leads to much larger values of $\kappa_{\text {raw }}$ in the subtropical gyre (not shown), exceeding $5000 \mathrm{~m}^{2} \mathrm{~s}^{-1}$. It appears that the seasonal cycle strongly affects the estimated thickness diffusivity in this region. We also note in passing that choosing different reference pressure levels for potential density (or $b$ ), on the other hand, changes the results shown here only very little (not shown).

Approaching the equator, $\kappa_{\text {raw }}$ remains large near the western boundary but changes its sign several times. Also, in the Gulf Stream region, there is a large area with negative values less than $-5000 \mathrm{~m}^{2} \mathrm{~s}^{-1}$, followed by changes in sign farther downstream in the North Atlantic Current. The appearance of large negative values may come as a surprise since we expect the Gulf Stream and North Atlantic Current to be baroclinically unstable regions where the available potential energy of the mean state is being released, and $\kappa$ in general should therefore be positive. To give insight into this issue, Fig. 4 shows the vertical eddy buoyancy flux $\overline{\left(w^{\prime} b^{\prime}\right)}$ in the main thermocline as an indicator for local energy transfer from mean potential energy to eddy kinetic energy by baroclinic instability (Lorenz, 1955). Over large areas, $\overline{w^{\prime} b^{\prime}}$ is positive, denoting indeed release of mean potential energy to eddy kinetic energy, as we expect. However, in the region immediately to the south of the Gulf Stream and farther downstream, and a few degrees latitude north and south of the equator [consistent with recent analysis of eddy feedbacks on equatorial currents by Jochum and MalanotteRizzoli (2004)], $\overline{w^{\prime} b^{\prime}}$ is negative with large magnitudes, pointing to the opposite mechanism. The regions with negative $\overline{w^{\prime} b^{\prime}}$, that is, regions with transfer from eddy kinetic energy to the mean stratification, are coinciding roughly with regions of negative $\kappa_{\text {raw }}$. Thus, negative $\kappa_{\text {raw }}$ s appear to be related to regions in which energy contained in mesoscale activity is feeding back to the mean flow.

The picture is complemented by a look at $\nu_{\text {raw }}$ shown in Fig. 3b. Note that $\nu_{\text {raw }}$ should vanish or at least be small for the downgradient GM parameterization to be valid (using the raw eddy fluxes). It is not; in fact, it is 
a)

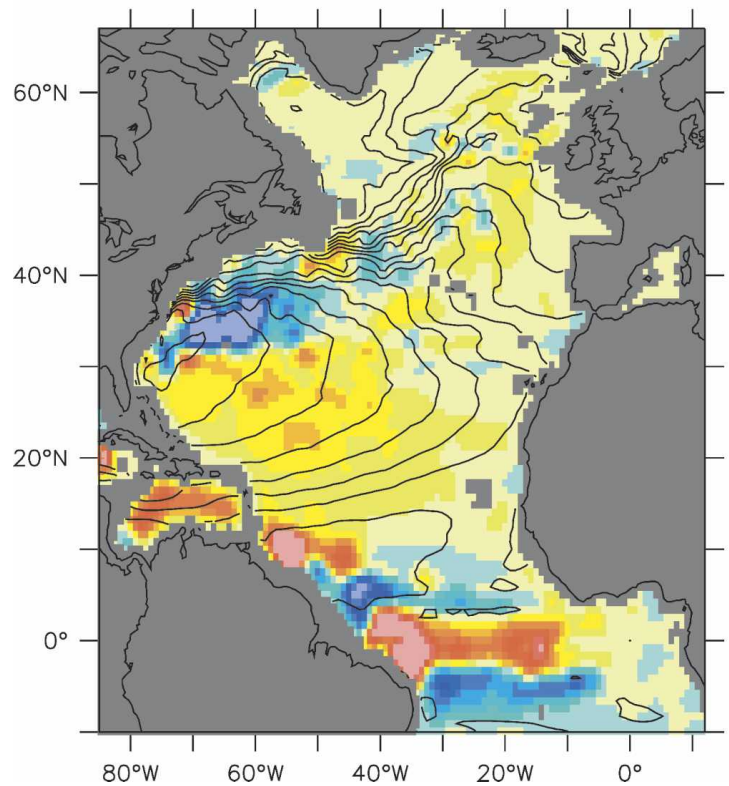

b)

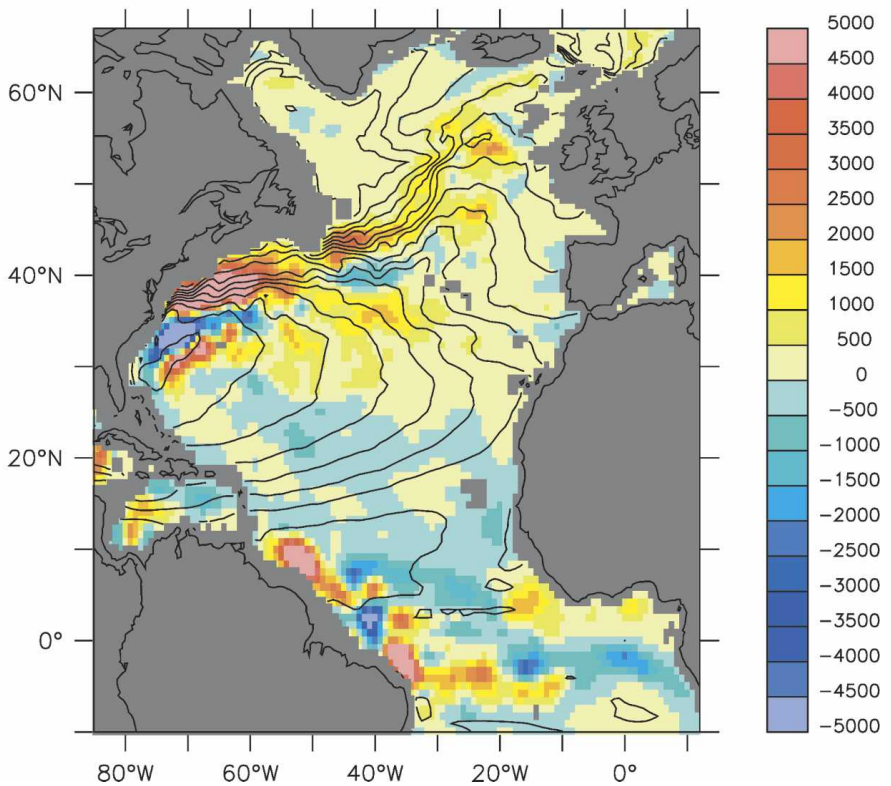

FIG. 3. The (a) $\kappa_{\text {raw }}$ and (b) $\nu_{\text {raw }}\left(\mathrm{m}^{2} \mathrm{~s}^{-1}\right)$ calculated from Eq. (21) using the raw horizontal eddy fluxes in 300-m depth. Also shown are contours of the mean buoyancy $\bar{b}$. Data are computed from 5-yr averages, and $\overline{\mathbf{u}_{h}^{\prime} b^{\prime}}$ and $\bar{b}$ are spatially averaged over 15 grid points. Calculated $\kappa_{\text {raw }}$ and $\nu_{\text {raw }}$ are also smoothed with a Hanning window over $40 \times 40$ grid points. Also shown are contours of the mean buoyancy $\bar{b}$.

of the same order of magnitude as $\kappa_{\text {raw }}$ and shows largescale gradients. A notable feature of $\nu_{\text {raw }}$ is the tendency for it to be positive in the northern half of the subtropical gyre and negative in the southern half of the subtropical gyre.

Equation (11) shows that $\nu$ acts in the quasigeostrophic approximation as a streamfunction that adds to the mean geostrophic flow and advects mean thickness (in contrast to diffusion of mean thickness related to $\kappa$ ). Figure 5 shows an estimate for the (geostrophic) streamfunction $\bar{\psi}$ of the mean flow, simply by solving $\nabla_{h}^{2} \bar{\psi}=\nabla_{h} \cdot \overline{\mathbf{u}}_{h}$ using $\overline{\mathbf{u}}$ from the model output. The values of $\bar{\psi}$ range about $\pm 50000 \mathrm{~m}^{2} \mathrm{~s}^{-1}$; thus, the $\nu$-related flow is on the order of $10 \%$ of the mean flow in our eddy-resolving model, which means that there is a significant eddy-induced part of the advection velocity. Note that assuming a uniform value of $\bar{\psi}$ over a layer with 1000-m thickness yields maximal transports of $\pm 50 \times 10^{6} \mathrm{~m}^{3} \mathrm{~s}^{-1}=50 \mathrm{~Sv}$, to which $\nu$ would contribute by more than $\pm 5 \mathrm{~Sv}$.

Now we substract rotational fluxes from the eddy fluxes F following Marshall and Shutts (1981) before projecting the fluxes onto $\nabla_{h} \bar{b}$. Figure 6 shows thickness diffusivities $\kappa_{\mathrm{MS}}$ and their counterpart $\nu_{\mathrm{MS}}$ estimated from Eq. (19) and Eq. (20). In large parts of the subtropical gyre, $\kappa_{\mathrm{MS}}$ has not changed much and is still positive and about $500-1500 \mathrm{~m}^{2} \mathrm{~s}^{-1}$. In the Gulf Stream region, on the other hand, the large region of negative $\kappa$ in Fig. 3 has been replaced by a region with large positive values of $\kappa$, although some negative values are still found. This comes along with positive values of $\nu_{\mathrm{MS}}$

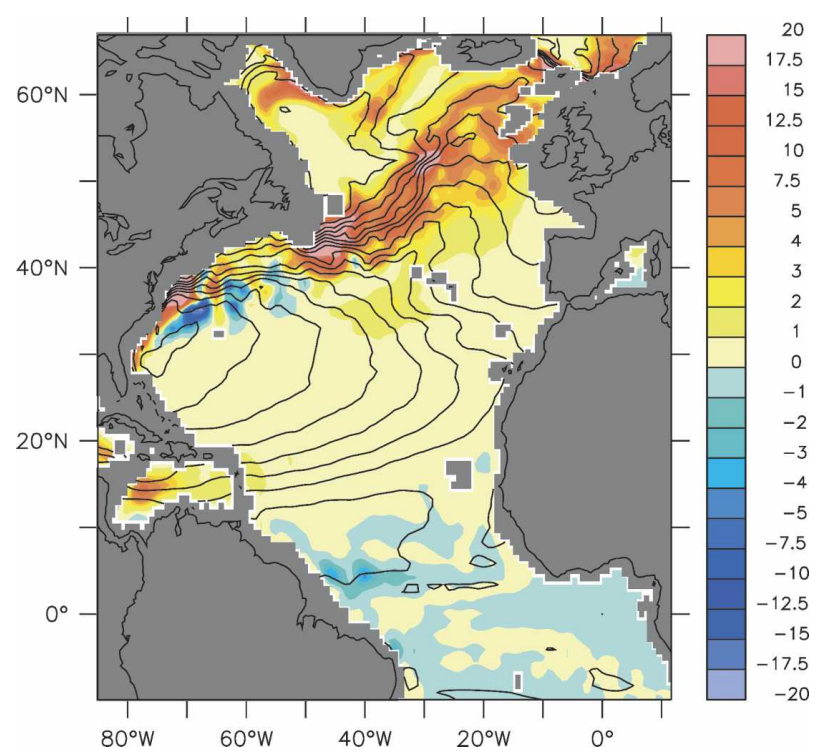

FIG. 4. Vertical eddy buoyancy flux $\overline{w^{\prime} b^{\prime}}$ in $300-\mathrm{m}$ depth $\left(10^{-9}\right.$ $\left.\mathrm{m}^{2} \mathrm{~s}^{-3}\right)$. Data are computed from 5 -yr averages, and $\overline{w^{\prime} b^{\prime}}$ is spatially averaged over $40 \times 40$ grid points. Also shown are contours of the mean buoyancy $\bar{b}$. 


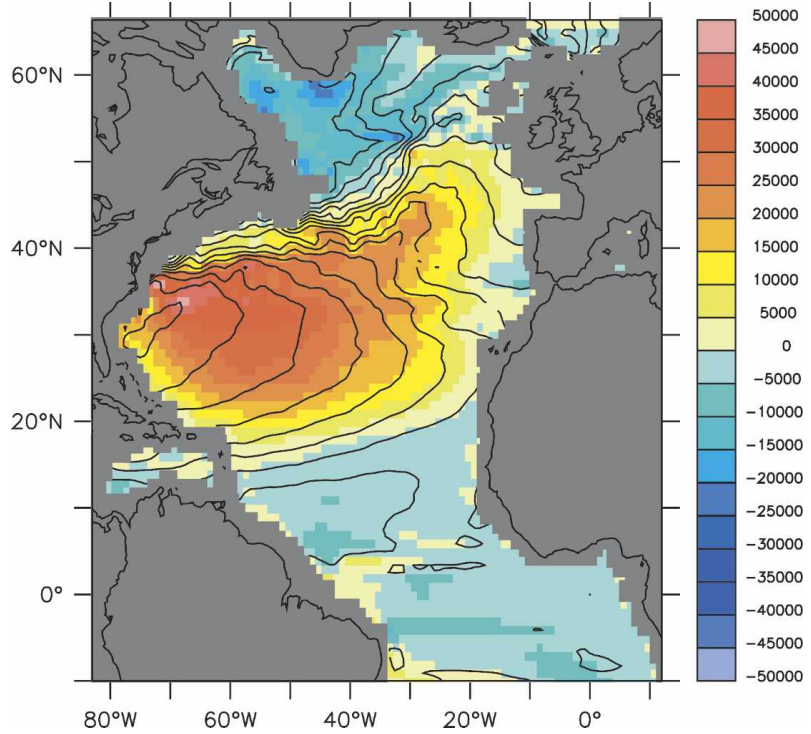

FIG. 5. The streamfunction $\Psi_{m}$ for the mean horizontal flow in 300-m depth (shaded; $\mathrm{m}^{2} \mathrm{~s}^{-1}$ ) estimated from $\nabla_{h}^{2} \Psi_{m}=\nabla_{h} \cdot \overline{\mathbf{u}}_{h}$. Also shown are contours of the mean buoyancy $\bar{b}$.

in the subtropical gyre. However, approaching the equator, $\kappa_{\mathrm{MS}}$ still tends to change sign and the pattern of $\kappa_{\mathrm{MS}}$ hardly changes when compared with $\kappa_{\text {raw }}$. We might argue that this is due to the geostrophic assump- tion in the approach by Marshall and Shutts (1981), a drawback which is relaxed using the definition for rotational eddy fluxes of Medvedev and Greatbatch (2004).

\section{Rotational eddy fluxes: Medvedev and Greatbatch (2004)}

Marshall and Shutts (1981) make several assumptions (e.g., that the mean flow is parallel to the mean buoyancy contours; see Fig. 5 for the validity of this assumption) and neglect several terms in the eddy variance equation (e.g., the triple-correlation term), which might play a more significant role than assumed, such that the results in terms of the estimated thickness diffusivity might be obscured. A generalization of this idea is given by Medvedev and Greatbatch (2004). Consider again the variance equation

$$
\frac{\partial}{\partial t} \bar{\phi}+\nabla_{h} \cdot \overline{\mathbf{u}_{h} \phi}+\mathbf{F}_{h} \cdot \nabla_{h} \bar{b}+R_{z} \overline{b^{\prime} w^{\prime}}=\overline{Q^{\prime} b^{\prime}},
$$

where $Q$ denotes diabatic forcing. We have again assumed the quasigeostrophic form of the buoyancy budget to derive the eddy variance budget, Eq. (23), a rather weak assumption given the strong stratification in the interior of the ocean. Now decompose the hori- a)

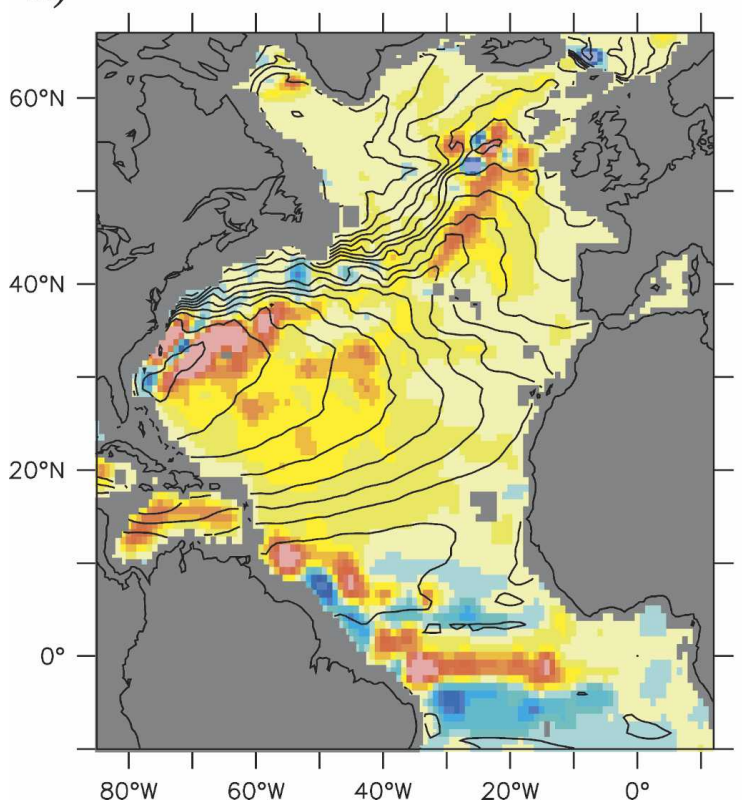

b)

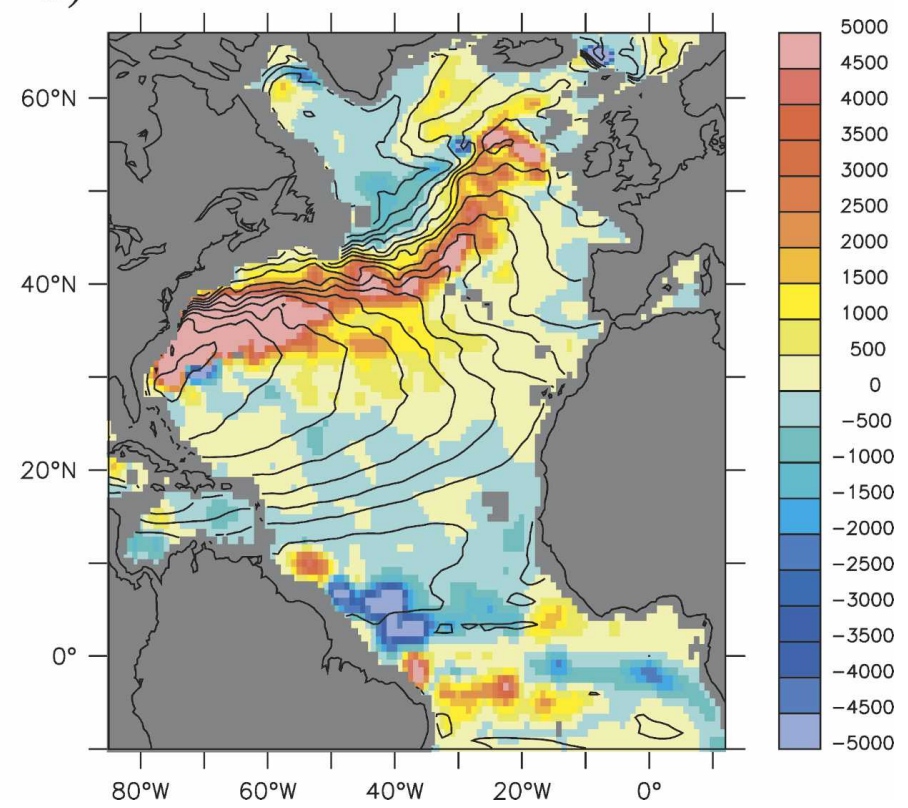

FIG. 6. The (a) $\kappa_{\mathrm{MS}}$ and (b) $\nu_{\mathrm{MS}}\left(\mathrm{m}^{2} \mathrm{~s}^{-1}\right)$ in 300-m depth estimated from horizontal eddy fluxes from which a rotational flux following Marshall and Shutts (1981) has been removed. Data are computed from 5-yr averages, and $\overline{\mathbf{u}_{h}^{\prime} b^{\prime}}$ and $\bar{b}$ are spatially averaged over 15 grid points. Calculated $\kappa_{\mathrm{MS}}$ and $\nu_{\mathrm{MS}}$ are also smoothed with a Hanning window over $40 \times 40$ grid points. Also shown are contours of the mean buoyancy $\bar{b}$. 
a)

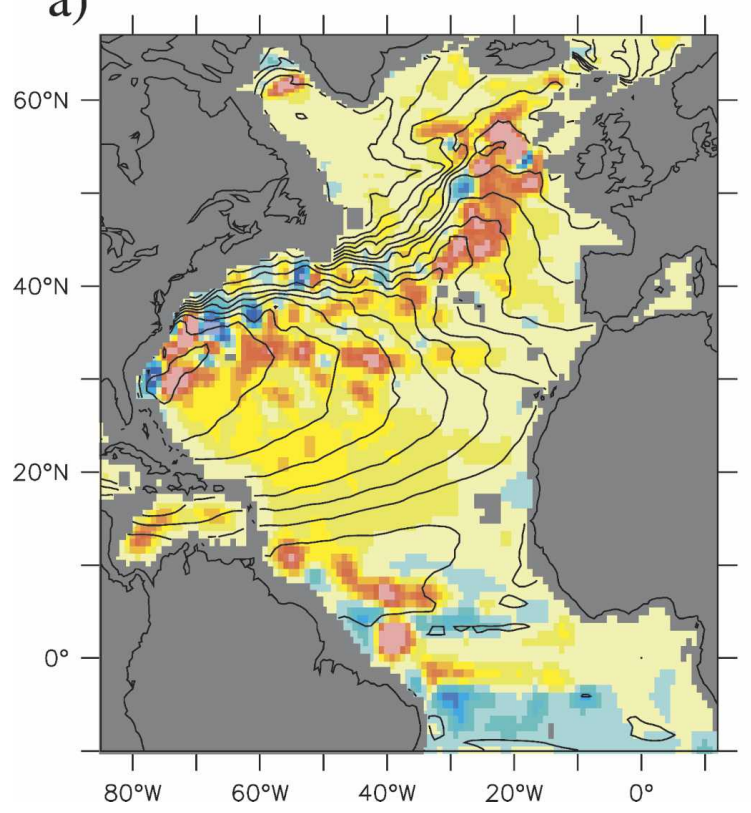

b)

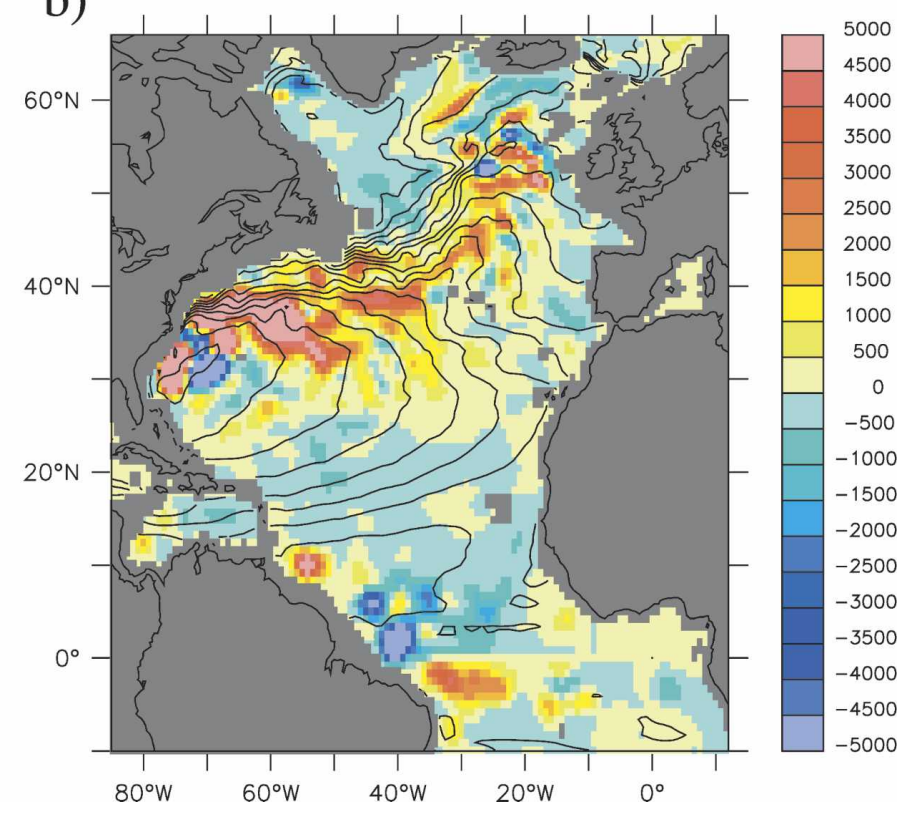

FIG. 7. The (a) $\kappa_{\mathrm{MG}}$ and (b) $\nu_{\mathrm{MG}}\left(\mathrm{m}^{2} \mathrm{~s}^{-1}\right)$ in 300-m depth estimated from eddy fluxes from which a rotational flux following Medvedev and Greatbatch (2004) has been removed. Data are computed from 5-yr averages, and $\overline{\mathbf{u}_{h}^{\prime} b^{\prime}}$ and $\bar{b}$ are spatially averaged over 15 grid points. Calculated $\kappa_{\mathrm{MS}}$ and $\nu_{\mathrm{MS}}$ are also smoothed with a Hanning window over $40 \times 40$ grid points. Also shown are contours of the mean buoyancy $\bar{b}$.

zontal advection of eddy variance into components along and across the horizontal gradient of the mean buoyancy; that is,

$$
\overline{\mathbf{u}_{h} \phi}=\alpha \nabla_{h} \bar{b}+\gamma \nabla \bar{p},
$$

and the eddy density flux as before as $\mathbf{F}_{h}=-\boldsymbol{\kappa}_{h} \bar{b}-$ $\nu \nabla \bar{b}+\nabla \theta$, which yields

$$
\begin{aligned}
\frac{\partial}{\partial t} \bar{\phi} & +\nabla_{h} \cdot \alpha \nabla_{h} \bar{b}+\underset{\gamma}{\nabla}(\theta-\gamma) \cdot \nabla_{h} \bar{b}+R_{z} \overline{b^{\prime} w^{\prime}}=\overline{Q^{\prime} b^{\prime}} \\
& +\kappa\left|\nabla_{h} b\right|^{2} .
\end{aligned}
$$

It is again straightforward to set $\theta=\gamma$. The difference from Marshall and Shutts (1981) is that no assumption about the nature of the mean flow was made; for example, the definition should be valid also for diabatic flows and at the equator. The thickness diffusivity $\kappa_{\mathrm{MG}}$ and its counterpart $\nu_{\mathrm{MG}}$ for the choice of the rotational flux potential of Medvedev and Greatbatch (2004) are thus defined by

$$
\begin{aligned}
& \kappa_{\mathrm{MG}}=-\left|\nabla_{h} \bar{b}\right|^{-2}\left(\overline{\boldsymbol{u}_{h}{ }_{h} b^{\prime}}-\underset{\urcorner}{\nabla} \theta_{\mathrm{MG}}\right) \cdot \nabla_{h} \bar{b} \quad \text { and } \\
& \nu_{\mathrm{MG}}=-\mid \nabla_{h} \bar{b}^{-2}\left(\overline{\mathbf{u}_{h}^{\prime}{ }^{\prime} b^{\prime}}-\nabla_{\urcorner} \theta_{\mathrm{MG}}\right) \cdot \nabla_{\urcorner} \bar{b}
\end{aligned}
$$

with $\theta_{\mathrm{MG}}=\left|\nabla_{h} \bar{b}\right|^{-2} \overline{\mathbf{u}_{h} \phi} \cdot \nabla \bar{b}$, which follows from Eq. (24). Note that a consequent application of the approach by Marshall and Shutts (1981) and Medvedev and Greatbatch (2004), using the variance flux decomposition $\overline{\mathbf{u}_{h} \boldsymbol{\phi}}=\alpha \boldsymbol{\nabla}_{h} \bar{b}+\gamma \boldsymbol{\nabla} \bar{b}+\boldsymbol{\nabla} \beta$ and the consideration of higher-order moments $\overline{b^{\prime n}}$, leads to the TRM-G version of Eden et al. (2007), that is, to Eq. (13). However, this expression is not used in the present paper. Note also that the three-dimensional rotational eddy flux in Eq. (2) is simply given by $\boldsymbol{\theta}=\left(0,0,-\theta_{\mathrm{MG}}\right)^{\mathrm{T}}$, and that $\left(\boldsymbol{\nabla} \times \boldsymbol{\theta}_{\mathrm{MG}}\right)_{h}=\boldsymbol{\nabla} \gamma$.

Figure 7 shows thickness diffusivities $\kappa_{\mathrm{MG}}$ and the streamfunction $\nu_{\mathrm{MG}}$ estimated from Eq. (26) and Eq. (27). Now there are slightly more grid points with negative values of $\kappa$ in the Gulf Stream region and the equatorial Atlantic in comparison with Fig. 6 and Fig. 3, although the regions with negative $\kappa_{\mathrm{MG}}$ continue to coincide with regions in which $\overline{w^{\prime} b^{\prime}}$ is negative, as we expect. On the other hand, positive values of $\kappa$ at the eastern flank of the North Atlantic Current have increased. Interestingly, the $\nu_{\mathrm{MG}}$ field continues to show the asymmetry between positive (negative) values over the northern (southern) part of the subtropical gyre. Indeed, the large-scale pattern of $\nu$ is much less affected by different choices of the rotational flux (cf. Fig. 3b, Fig. 6b, and Fig. 7b) than is the pattern of $\kappa$.

Figure 8 shows the vertical dependency of the three different estimates for $\kappa$ on a meridional section along $50^{\circ} \mathrm{W}$. As already seen in Fig. $3, \kappa_{\text {raw }}$ tends to show large negative values in the vicinity of strong boundary currents, that is, in Fig. 8 a between $5^{\circ}$ and $10^{\circ} \mathrm{N}$. As 
a)

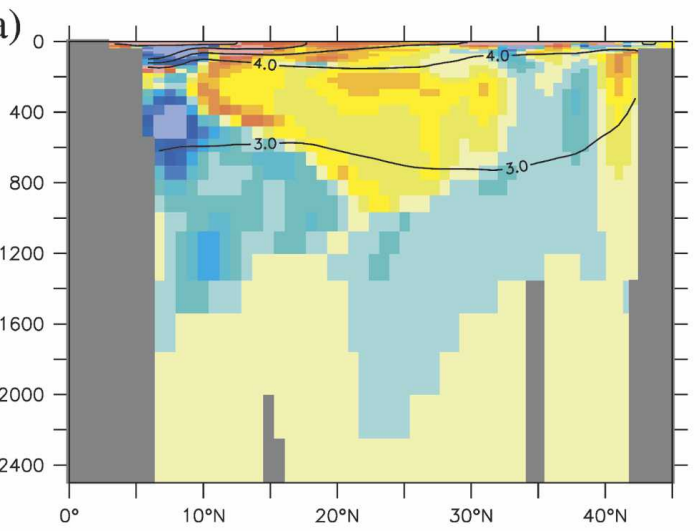

c)

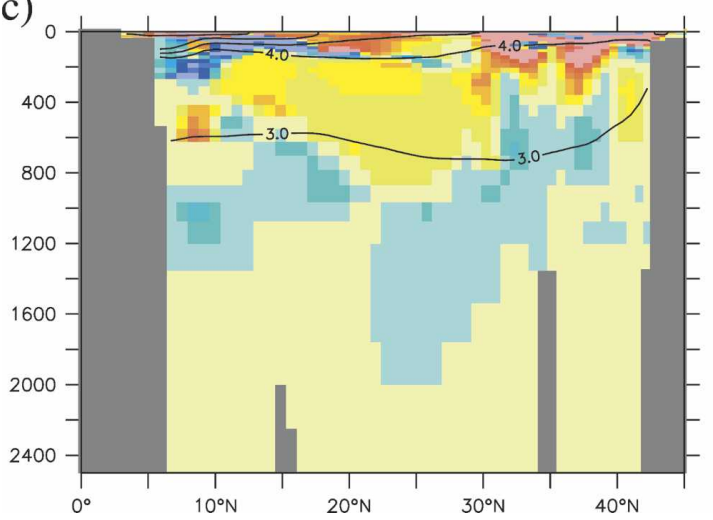

b)

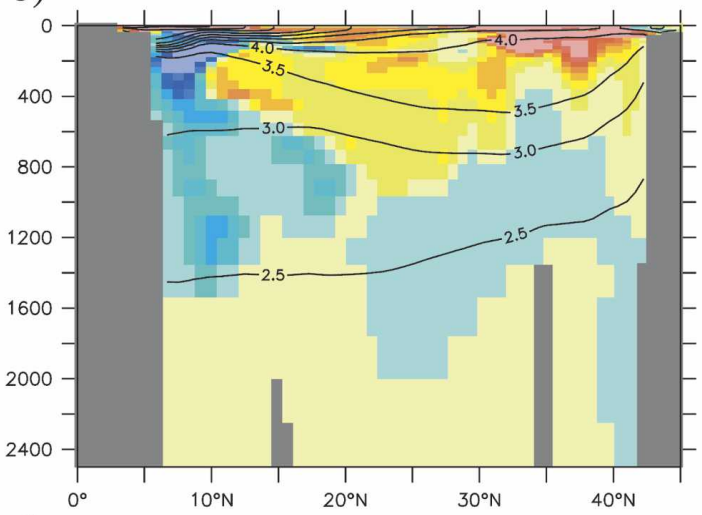

d)

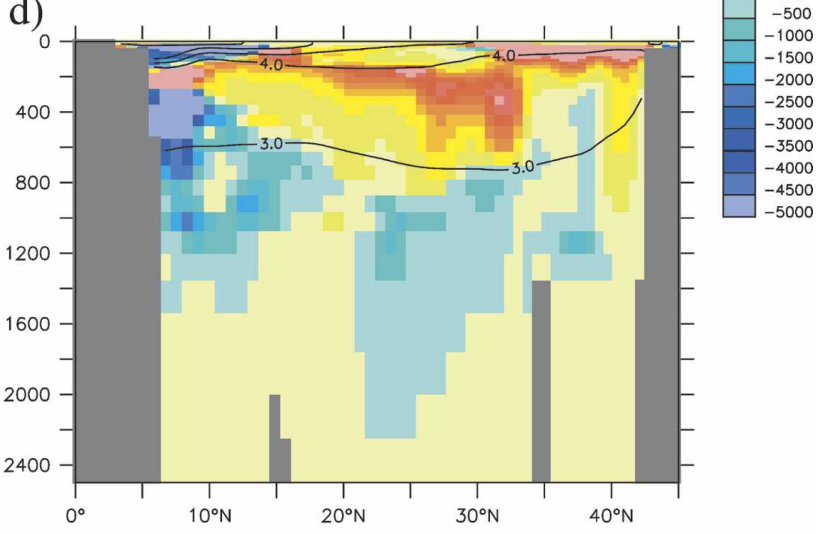

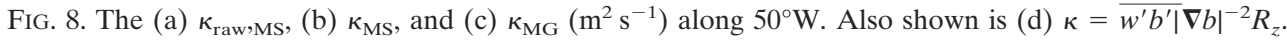

before, these negative values tend to get smaller, removing meaningful rotational fluxes for the case $\kappa_{\mathrm{MS}}$ and $\kappa_{\mathrm{MG}}$ (Figs. $8 \mathrm{~b}$ and $8 \mathrm{c}$ ). On the other hand, the vertical structure of the thickness diffusivity is similar in all estimates, that is, $\kappa$ tends to be larger in the thermocline and above and lower, almost zero, below $\sim 1000 \mathrm{~m}$. A similar vertical structure can be seen for the thickness advection streamfunction $\nu$ (not shown) and at other meridional sections. Note also that the small negative values of $\kappa$ in Figs. $8 \mathrm{~b}$ and $8 \mathrm{c}$ below $\sim 1000 \mathrm{~m}$ coincide with regions in which $\overline{w^{\prime} b^{\prime}}$ is negative as before for the horizontal structure of $\kappa$ in Fig. 6 and Fig. 7. Figure $8 \mathrm{~d}$ shows $\kappa=R_{z} \overline{w^{\prime} b^{\prime}}\left|\nabla_{h} \bar{b}\right|^{-2}$, as deduced from the eddy variance budget Eq. (16) and as the leading-order term in Eq. (13) to illustrate this point.

\section{Optimized rotational eddy fluxes}

As an alternative to specifying a rotational flux based on theoretical considerations, we now proceed to estimate a rotational flux by minimizing numerically $\kappa$ and $\nu$ and $\theta_{3}$ in

$$
\begin{gathered}
\int d V \operatorname{var}(\kappa)^{-1}\left(\kappa-\kappa_{0}\right)^{2}+\operatorname{var}(\nu)^{-1}\left(\nu-v_{0}\right)^{2} \\
+\operatorname{var}\left(\theta_{3}\right)^{-1}\left(\theta_{3}-\theta_{0}\right)^{2}=\min ,
\end{gathered}
$$

under the constraint that $\kappa, \nu$, and $\theta_{3}$ satisfy $\mathbf{F}_{h}=$ $-\kappa \nabla_{h} \bar{b}-\nu \nabla \bar{b}+\nabla \theta_{3}$ using standard methods (Wunsch 1996). Since we try to solve an underdetermined system, there are an infinite number of solutions satisfying the flux decomposition; therefore, deviations from a priori parameters $\kappa_{0}, \nu_{0}$, and $\theta_{0}$ are minimized under the constraint that the parameters are satisfying the flux decomposition. Furthermore, the system is weighted by specifying deviations over which the parameters are allowed to vary, that is, $\operatorname{var}(\kappa, \nu)$ and $\operatorname{var}\left(\theta_{3}\right)$.

It is important to formulate explicitly the question asked by the minimization, Eq. (28), to interpret the answer that is obtained. We ask for a decomposition of the horizontal eddy fluxes in terms of the parameters $\kappa$, $\nu$, and $\theta_{3}$, which gives minimal deviations of the parameters from a priori known values. We do not know much about the a priori parameters. What we do know is that $\kappa$ should be positive in an integral sense, as given from the balance of available potential energy in the 
TABLE 1. Parameter for the different minimizations discussed in section 6. Values for $\kappa$ and $\nu$ are given in meters squared per second while values for $\theta$ are given in cubic meters per second.

\begin{tabular}{lccccc}
\hline \hline \multicolumn{1}{c}{ Expt } & $\kappa_{0}$ & $\nu_{0}$ & $\theta_{0}$ & $\operatorname{var}(\kappa, v)^{1 / 2}$ & $\operatorname{var}\left(\theta_{3}\right)^{1 / 2}$ \\
\hline MINI-I & $2 \times 10^{3}$ & 0 & $-\gamma$ & $10^{3}$ & $10^{4}$ \\
MINI-II & 0 & 0 & $-\gamma$ & $10^{3}$ & $10^{4}$ \\
MINI-III & 0 & 0 & 0 & $10^{3}$ & $10^{4}$ \\
\hline
\end{tabular}

quasigeostrophic approximation, and that $\theta_{3}$ could be chosen using Marshall and Shutts (1981) or Medvedev and Greatbatch (2004) as a guide. Note that it also appears possible to minimize spatial variations of the parameters as well, introducing terms like $\left|\nabla_{h} \kappa\right|^{2}$ in Eq. (28), sometimes called a "beautifier," as fields are getting smoother in this case. However, in our experience such a beautifier gives similar results as to smooth the field afterward, such that we stick to the simplest form, Eq. (28).

We start by showing results of a first minimization (MINI-I) using $\kappa_{0}=2000 \mathrm{~m}^{2} \mathrm{~s}^{-1}, \nu_{0}=0$, and $\theta_{0}=-\gamma$ as a priori parameters and using the weights $\operatorname{var}(\kappa, \nu)^{1 / 2}=$ $1000 \mathrm{~m}^{2} \mathrm{~s}^{-1}$ and $\operatorname{var}\left(\theta_{3}\right)^{1 / 2}=1000 \mathrm{~m}^{3} \mathrm{~s}^{-3}$. Note that all parameters of the different minimizations discussed here are summarized in Table 1 . In other words, we use as a priori information that $\kappa$ should be similar to the canonical, constant value used in coarse-resolution models and that there are rotational eddy fluxes pres- ent in the "raw" fluxes as given by the choice of Medvedev and Greatbatch (2004). We minimize deviations of $\kappa$ from the canonical value, implying that we search for a (more or less) positive, constant value of $\kappa$. For $\nu$ we simply search for small values, implying that this parameter should be zero. In effect, this choice for the a priori parameters implements some of the currently accepted knowledge about eddy fluxes.

Figure 9 shows the results of the minimization MINI-I in terms of the optimal $\kappa$ and $\nu$. As constrained by the minimal condition, the optimal $\kappa$ is now positive over large regions of the North Atlantic. However, in a rather larger area near the Gulf Stream separation and $5^{\circ}$ north and south of the equator we find negative values of the thickness diffusivity. Note that these regions still coincide with the regions where $\overline{w^{\prime} b^{\prime}}$ is negative. The positive values in the subtropical North Atlantic are similar to those before (e.g., Fig. 7); overall the optimal $\kappa$ ranges between 500 and $1500 \mathrm{~m}^{2} \mathrm{~s}^{-1}$. The values of the optimal $\nu$ have only slightly changed as well; they are still of the same order of magnitude as $\kappa$. Note that we aimed to minimize values of $\nu$ in the solution of Eq. (28). Interestingly, $v$ remains predominantly positive north of about $30^{\circ} \mathrm{N}$ and negative south of this latitude, as we noted in the other cases. Indeed, this asymmetry in $\nu$ is a robust feature of our results. We take this as evidence that the $\nu$-related part of the bolus velocity is an important aspect of eddy fluxes. a)

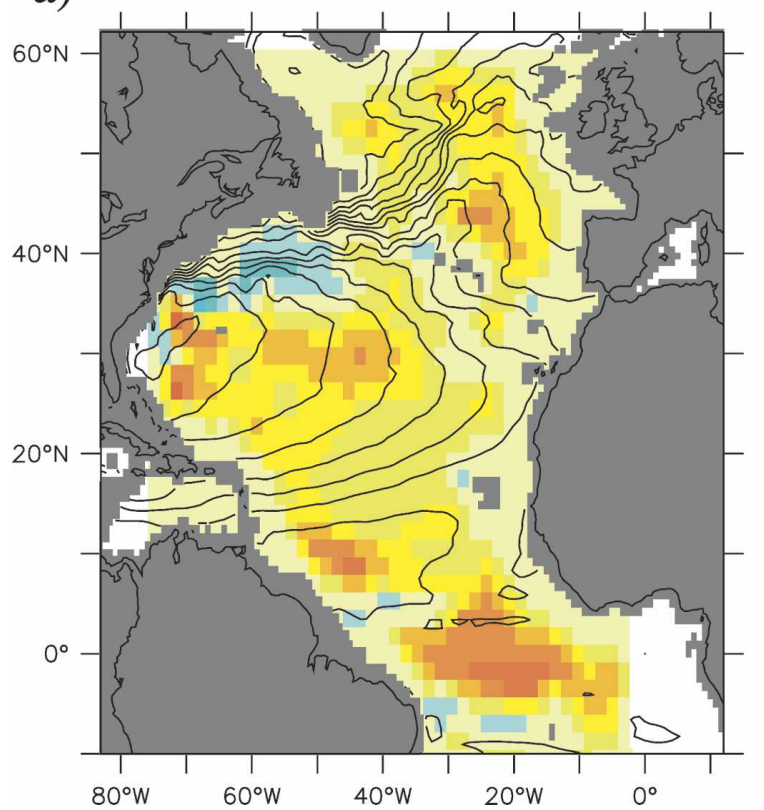

b)

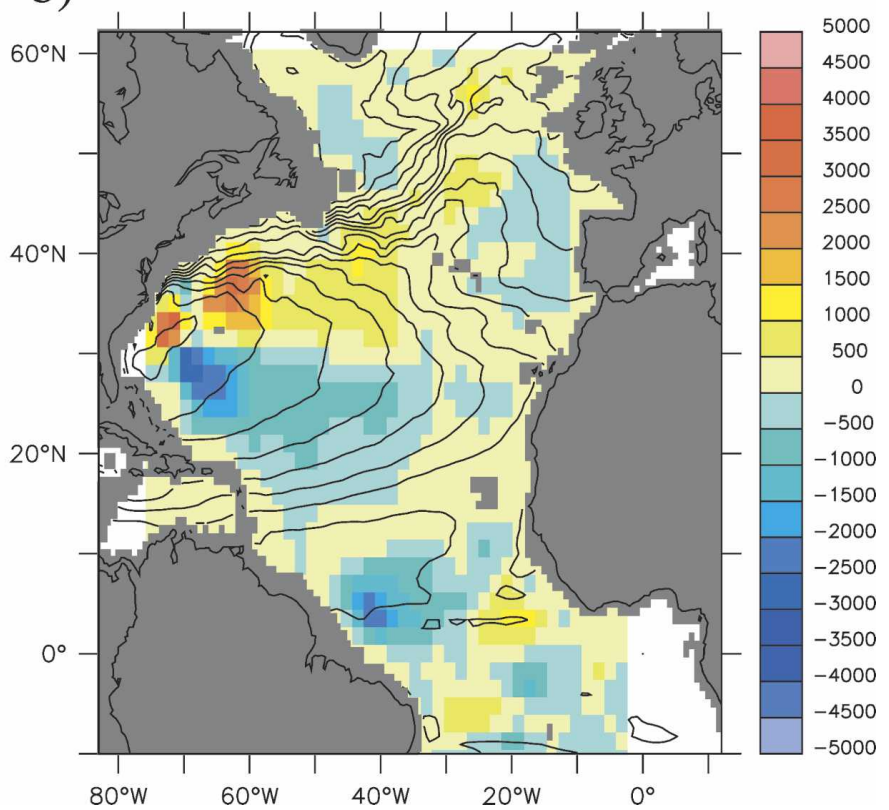

FIG. 9. The (a) $\kappa$ and (b) $\nu\left(\mathrm{m}^{2} \mathrm{~s}^{-1}\right)$ in 300-m depth estimated from eddy fluxes from which an optimized rotational flux has been removed (expt MINI-I, see text for details). Also shown are contours of the mean buoyancy $\bar{b}$. 
a)

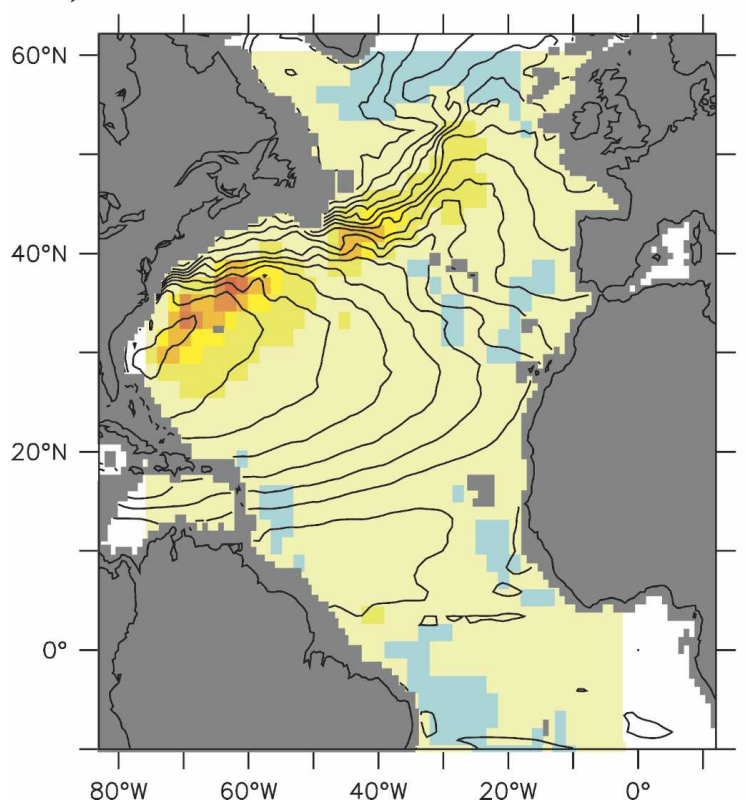

b)

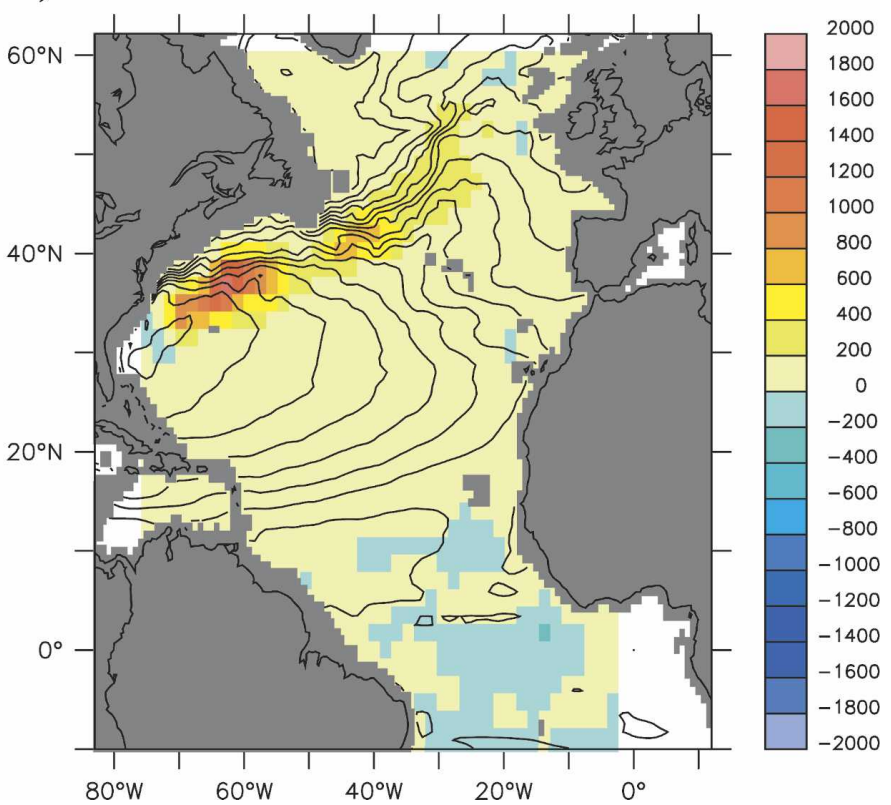

FIG. 10. The optimal rotational gauge potential (a) $\theta_{3}$ and (b) $-\gamma\left(\mathrm{m}^{3} \mathrm{~s}^{-3}\right)$ in $300-\mathrm{m}$ depth (expt MINI-I; see text for details). Also shown are contours of the mean buoyancy $\bar{b}$.

Figure 10 displays the optimal rotational gauge potential $\theta_{3}$ and also $-\gamma$, which was used to estimate $\kappa$ and $\nu$ in Fig. 7 and serves as the a priori information for $\theta_{3}$ in MINI-I. Both figures are rather similar. Largest gradients of similar direction and amplitude show up in both the a priori and a posteriori rotational potential in Fig. 10 in the Gulf Stream region and farther downstream, pointing toward strong westward rotational eddy fluxes in this region.

We show in Fig. 11 results from a second minimization (MINI-II) using the a priori parameter $\kappa_{0}=0$, while all other parameters stay the same as in MINI-I. Note that $\nu$ (and $\theta_{3}$, not shown) hardly changes, displaying again a similar pattern as in all figures before. By construction, values of $\kappa$ are decreasing in this minimization. In consequence, there are now larger regions with negative values of $\kappa$. However, the magnitude of the negative values is less than before for $\kappa$ in the flux decompositions by Marshall and Shutts (1981) or Medvedev and Greatbatch (2004). Only near the coast of North America at the Gulf Stream separation point few values more negative than $-500 \mathrm{~m}^{2} \mathrm{~s}^{-1}$ appear. Note that the regions of negative $\kappa$ still coincide with regions of negative $\overline{w^{\prime} b^{\prime}}$. On the other hand, positive values of $\kappa$ are dominating even in this flux decomposition such that the integral constraint on $\kappa$ to be positive will be satisfied.

In a third minimization (MINI-III), we also set $\theta_{0}=$ 0 , while all other parameters stay the same as in MINI-
II. The results in terms of $\kappa, \nu$, and $\theta_{3}$ (not shown) are very similar to MINI-II, showing that the choice for the rotational eddy fluxes by Medvedev and Greatbatch (2004) yields similar results as the outcome from our minimization constraint by the a priori information of zero rotational eddy flux, pointing toward the need of rotational eddy fluxes.

From the similarity of the large-scale patterns in $\kappa, \nu$, and $\theta_{3}$ in the minimization and the above sections, we conclude that the previous flux decompositions are in accordance with the results from the minimization. This means that there are no artificially large parameters of some kind in the previous flux decompositions. In particular, the similar patterns of $\nu$ in all results and the fact that $\nu$ does not vanish in the minimization, denotes that $\nu$ is a fundamental aspect of eddy fluxes and is actually needed in a consistent flux decomposition. The same holds for the rotational eddy fluxes.

\section{Conclusions}

Previous attempts to verify the GM parameterization for mesoscale eddy transport, and to obtain an estimate for the thickness diffusivity, have been thwarted by the presence of large rotational eddy tracer fluxes that have no effect on the mean buoyancy budget, but complicate the interpretation of the individual eddy tracer fluxes. Here, we have used long-term-averaged output from a $1 / 12{ }^{\circ}$ eddy-resolving model of the North Atlantic Ocean 
a)

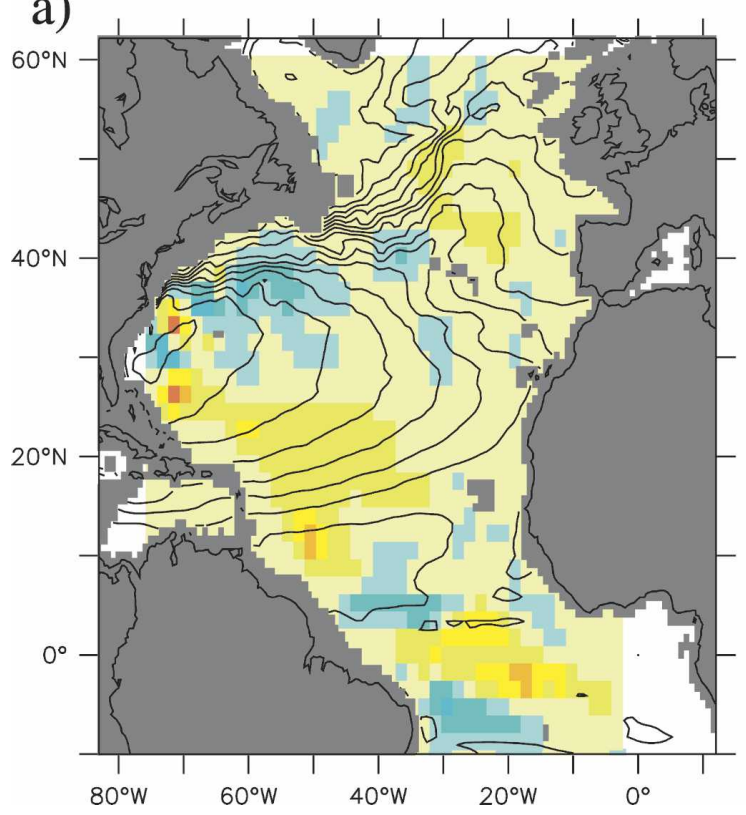

b)

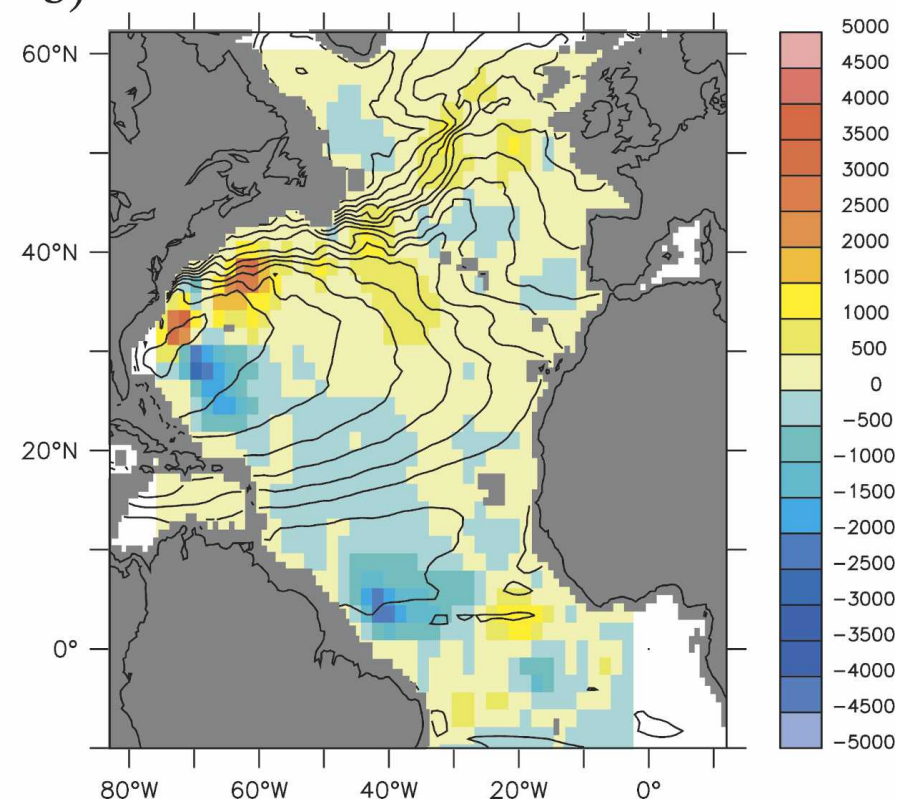

FIG. 11. The (a) $\kappa$ and (b) $\nu\left(\mathrm{m}^{2} \mathrm{~s}^{-1}\right)$ in 300-m depth estimated from eddy fluxes from which an optimized rotational flux has been removed (expt MINI-II; see text for details). Also shown are contours of the mean buoyancy $\bar{b}$.

to estimate values for the thickness diffusivity $\kappa$ and its related parameter $v$ using different procedures to remove a rotational flux prior to the estimation.

Rotational fluxes do not appear in the flux divergence and, hence, do not change the density budget. Using different rotational fluxes leads to different diagnoses for $\kappa$ and $\nu$; but it should be noted that as the divergence of the different flux decompositions is the same in all cases, the effect in the mean density budget for all flux decompositions is also always the same.

The point of choosing a rotational flux is to ensure that the $\kappa$ and $\nu$ that result have a clear and simple physical interpretation. This point is nicely illustrated by Eq. (16) where choosing the rotational flux $\theta=\gamma$ immediately shows that the diffusivity $\kappa$ is proportional to $\overline{w^{\prime} b^{\prime}}$ (which is positive when available potential energy is being released).

\section{a. Thickness diffusivity}

Using the raw fluxes (no rotational flux removed), large negatives values of $\kappa$ are diagnosed, particularly in the Gulf Stream region, farther downstream in the North Atlantic Current, and in the equatorial Atlantic. Removing a rotational flux based on the suggestion of Marshall and Shutts (1981), as in Marshall (1984), or on the more general theory of Medvedev and Greatbatch (2004) leads to smaller regions of negative thickness diffusivities and smaller magnitudes of the negative val- ues, but they are still present. These regions coincide with regions of energy transfer from eddy kinetic energy to the mean potential energy.

In the subtropical North Atlantic (positive) values for $\kappa$ estimated from the raw fluxes are from 500 to $1500 \mathrm{~m}^{2} \mathrm{~s}^{-1}$ in the main thermocline over wide areas. These values are similar to previous estimates by Rix and Willebrand (1996) and Jochum (1997) using similar models at lower (eddy permitting) resolution. It appears that higher model resolution does not effect the estimation of thickness diffusivity much. In all estimates, the vertical structure of $\kappa$ is similar, that is, high $\left(500-2000 \mathrm{~m}^{2} \mathrm{~s}^{-1}\right)$ in the main thermocline and above and lower, almost zero, below $\sim 1000 \mathrm{~m}$, again coinciding with the sign of the energy transfer from eddy kinetic energy to the mean potential energy, which is negative below the thermocline.

As an alternative to specifying a rotational flux based on theoretical considerations, we also used an optimization technique to estimate the rotational flux similar to Rix and Willebrand (1996). In this case, the absolute value of $\kappa$ can be influenced by a priori information and the choice of the cost function by construction: since we try to obtain locally three parameters $(\kappa, \nu$, and $\theta)$ having only two independent parameters $\left(\overline{u^{\prime} b^{\prime}}\right.$ and $\left.\overline{v^{\prime} b^{\prime}}\right)$, the problem is underdetermined. We have implemented what is known as a priori information and have chosen a standard choice for the formulation of the cost function. We obtain higher (lower) thickness diffusivi- 
ties in the subtropical gyre by constraining the solution to higher (smaller) a priori values of $\kappa$. However, negative $\kappa$ tends to be always present in regions where energy transfer from eddy kinetic energy to the mean potential energy occurs, consistent with the previous estimates. Consequently, the rotational fluxes do not deviate much from a priori values given by the theoretical considerations.

In conclusion, all of our estimates show large horizontal variations in the values of the thickness diffusivity and consistently low or negative values of $\kappa$ in regions where eddies are feeding the mean flow. A constant value of $1000 \mathrm{~m}^{2} \mathrm{~s}^{-1}$ for $\kappa$ as widely used in noneddy-resolving models appears therefore to be only a very rough first-order approximation to this spatial dependence of thickness diffusivity. A local downgradient parameterization for thickness diffusivity, as anticipated by Gent and McWilliams (1990), might have to take negative values of $\kappa$ in certain regions into account (Berloff, 2005), although numerical restrictions on negative diffusivities might complicate the implementation in models.

\section{$b$. The v-related part of the fluxes}

We began by developing the theoretical basis for the GM parameterization within the framework of the transformed Eulerian mean. The analysis reveals a previously neglected aspect of the bolus velocity that is associated with the horizontal flux of buoyancy along, rather than across, the mean buoyancy contours. This part of the flux can be interpreted as eddy-induced advection rather than diffusion of mean isopycnal thickness (related to $\kappa$ ) indicating when the lateral eddy density fluxes cannot be described by isotropic diffusion only. It should be stressed, however, that both eddyinduced diffusion and advection of isopycnal thickness contribute to the three-dimensional bolus velocity appropriate to the GM parameterization; that is, both parts act as advection of density in three dimensions.

Since $\nu$ does not figure in the balance of available potential energy, there is no integral constraint on the sign of $\nu$, in contrast to its counterpart the thickness diffusivity $\kappa$ which has to be positive in an integral sense. It has been shown that $\nu$ acts as a streamfunction for advection of mean thickness. Comparing $\nu$ with an estimate of the mean geostrophic streamfunction, which also advects mean isopycnal thickness, shows that $\nu$ is on the order of $10 \%$ of the mean flow in midlatitudes and on the same order of magnitude in the Tropics.

The $\nu$-related part of the eddy flux shows up when the lateral eddy fluxes cannot be described by isotropic diffusion only, pointing toward the need of anisotropic lateral mixing. Such an anisotropy has, indeed, been found in observations [see, for example, Stammer and Böning (1996) for a review], and thus it appears obvious, in retrospect, that the parameter $\nu$ becomes important in the present diagnosis. The large-scale spatial pattern of $\nu$ is quite robust for all different choices of rotational fluxes that have been considered here. It is speculated that a possible source for anisotropic lateral mixing is given by the $\beta$ effect (Rhines 1977) on quasigeostrophic turbulence. Westward advection of mean thickness in the subtropical gyre, as implied by the large-scale pattern of $\nu$ in all estimates, might be related to the $\beta$ effect, reminiscent of long baroclinic Rossby waves while, for instance, northeastward advection in the North Atlantic Current is perhaps associated with the advection of eddies by the mean flow.

For the GM parameterization to be valid, the $\nu$-related part of the flux should be zero or small compared to $\kappa$. In contrast, all of our estimates show values of $\nu$ comparable or larger than $\kappa$. In particular, that $\nu$ does not vanish in the minimization denotes that $\nu$ is a fundamental aspect of eddy fluxes and is actually needed for a consistent flux parameterization. This previously neglected aspect of eddy tracer fluxes demands attention in parameterizing impacts of mesoscale activity on tracers in non-eddy-resolving models.

Acknowledgments. The model integrations have been performed on an NEX-SX8 at the computing center of the University Kiel, Germany, and on an NECSX6 at the Deutsches Klimarechenzentrum (DKRZ), Hamburg, Germany. RJG is grateful for funding support from NSERC and CFCAS through the NSERC Discovery Grant program and the Canadian CLIVAR Research Network. We also thank an anonymous reviewer and J. Marshall for helpful comments.

\section{REFERENCES}

Allen, J. S., P. R. Gent, and D. D. Holm, 1997: On Kelvin waves in balanced models. J. Phys. Oceanogr., 27, 2060-2063.

Andrews, D. G., and M. E. McIntyre, 1976: Planetary waves in horizontal and vertical shear: The generalized Eliassen-Palm relation and the zonal mean acceleration. J. Atmos. Sci., 33, 2031-2048.

Berloff, P., 2005: Random-forcing model of the mesoscale oceanic eddies. J. Fluid Mech., 529, 71-95.

Brandt, P., A. Funk, L. Czeschel, C. Eden, and C. Böning, 2007: Ventilation and transformation of Labrador Sea Water and its rapid export in the Deep Labrador Current. J. Phys. Oceanogr., in press.

Bryan, K., J. K. Dukowicz, and R. D. Smith, 1999: On the mixing coefficient in the parameterization of bolus velocity. J. Phys. Oceanogr., 29, 2442-2456.

Dengler, M., F. A. Schott, C. Eden, P. Brandt, J. Fischer, and R. J. Zantopp, 2004: Break-up of the Atlantic deep western boundary current into eddies at $8^{\circ} \mathrm{S}$. Nature, 432, 1018-1020. 
Drijfhout, S. S., and W. Hazeleger, 2001: Eddy mixing of potential vorticity versus thickness in an isopycnic ocean model. $J$. Phys. Oceanogr., 31, 481-505.

Eden, C., and C. W. Böning, 2002: Sources of eddy kinetic energy in the Labrador Sea. J. Phys. Oceanogr., 32, 3346-3363.

— , R. J. Greatbatch, and D. Olbers, 2007: Interpreting eddy fluxes. J. Phys. Oceanogr., in press.

Fox-Kemper, B., R. Ferrari, and J. Pedlosky, 2003: On the indeterminacy of rotational and divergent eddy fluxes. J. Phys. Oceanogr., 33, 478-483.

Gent, P. R., and J. C. McWilliams, 1990: Isopycnal mixing in ocean circulation models. J. Phys. Oceanogr., 20, 150-155.

_ J. J. Willebrand, T. J. McDougall, and J. C. McWilliams, 1995: Parameterizing eddy-induced tracer transports in ocean circulation models. J. Phys. Oceanogr., 25, 463-474.

Jayne, S. R., and J. Marotzke, 2002: The oceanic eddy heat transport. J. Phys. Oceanogr., 32, 3328-3345.

Jochum, M., 1997: Eine ortsabhängige Parameterisierung von Wirbelflüssen. M.S. thesis, Theoretische Ozeanographie, Institut für Meereskunde, $62 \mathrm{pp}$.

- , and P. Malanotte-Rizzoli, 2004: A new theory for the generation of equatorial subsurface countercurrents. J. Phys. Oceanogr., 34, 755-771.

Lorenz, E. N., 1955: Available potential energy and the maintenance of the general circulation. Tellus, 7, 157-167.

Marshall, D., 1984: Eddy-mean-flow interaction in a barotropic ocean model. Quart. J. Roy. Meteor. Soc., 110, 573-590.

Marshall, J., and G. Shutts, 1981: A note on rotational and divergent eddy fluxes. J. Phys. Oceanogr., 11, 1677-1679.

McDougall, T. J., and P. C. McIntosh, 2001: The temporalresidual-mean velocity. Part II: Isopycnal interpretation and the tracer and momentum equations. J. Phys. Oceanogr., 31, 1222-1246.

Medvedev, A. S., and R. J. Greatbatch, 2004: On advection and diffusion in the mesosphere and lower thermosphere: The role of rotational fluxes. J. Geophys. Res., 109, D07104, doi:10.1029/2003JD003931.

Nakamura, M., and Y. Chao, 2000: On the eddy isopycnal thick- ness diffusivity of the Gent-McWilliams subgrid mixing parameterization. J. Climate, 13, 502-510.

Pacanowski, R. C., 1995: MOM 2 documentation, user's guide and reference manual. GFDL Ocean Group Tech. Rep. 3, 232 pp.

Peterson, K. A., and R. J. Greatbatch, 2001: Vorticity fluxes in shallow water ocean models. Atmos.-Ocean, 39, 1-14.

Radko, T., and J. Marshall, 2004: Eddy-induced diapycnal fluxes and their role in the maintenance of the thermocline. J. Phys. Oceanogr., 34, 372-383.

Rhines, P., 1977: The dynamics of unsteady currents. The Sea, E. D. Goldberg, Ed., Marine Modeling, Vol. 6, John Wiley and Sons, 189-318.

Rix, N., and J. Willebrand, 1996: A note on the parameterization of eddy-induced mixing from eddy-resolving model data. $J$. Phys. Oceanogr., 26, 2281-2285.

Roberts, M. J., and D. P. Marshall, 2000: On the validity of downgradient eddy closures in ocean models. J. Geophys. Res., 105 (C12), 28 613-28 627.

Smith, R. D., and P. R. Gent, 2004: Anisotropic Gent-McWilliams parameterization for ocean models. J. Phys. Oceanogr., 34, 2541-2564.

Solovev, M., P. H. Stone, and P. Malanotte-Rizzoli, 2002: Assessment of mesoscale eddy parameterizations for a single-basin coarse-resolution ocean model. J. Geophys. Res., 107, 3126, doi:10.1029/2001JC001032.

Stammer, D., and C. W. Böning, 1996: Generation and distribution of mesoscale eddies in the North Atlantic Ocean. The Warmwatersphere of the North Atlantic Ocean, W. Krauss, Ed., Gebrüger Bornträger, 159-193.

Stevens, D. P., 1990: On open boundary conditions for three dimensional primitive equation ocean circulation models. Geophys. Astrophys. Fluid Dyn., 51, 103-133.

Treguier, A. M., 1999: Evaluating eddy mixing coefficients from eddy-resolving ocean models: A case study. J. Mar. Res., 57, 89-108.

Wunsch, C., 1996: The Ocean Circulation Inverse Problem. Cambridge University Press, 458 pp. 